\title{
Horizontal mixing in lakes
}

\author{
F. Peeters, A. Wüest, G. Piepke, and D. M. Imboden \\ Swiss Federal Institute for Environmental Science and Technology and Swiss Federal Institute of \\ Technology, Dübendorf, Switzerland
}

\begin{abstract}
Horizontal mixing in the upper hypolimnion of lakes far from the boundaries was studied in lake basins with surface areas between 5 and $220 \mathrm{~km}^{2}$ by observing the growth of the concentration distribution of the fluorescent dye sodium fluorescein (uranin). In each of the eight experiments, between 0.2 and $2 \mathrm{~kg}$ of uranin was instantaneously released into the lake in the appropriate depth (between 15 and $25 \mathrm{~m}$ ) in such a way to keep the initial cloud size as small as possible. The horizontal extension of the cloud was repeatedly determined by integration of numerous vertical profiles. These surveys served to test theoretical models for horizontal mixing. The temporal development of the size and of the variances along the principal axes of the tracer concentration distribution was the main property considered here. The experiments cover a range of cloud sizes between $3 \times 10^{2}$ and $3 \times 10^{5} \mathrm{~m}^{2}$. None of them support the hypothesis that cloud size grows with elapsed time raised to the power of 3 as predicted by the inertial subrange turbulence model first applied to dispersion by Batchelor [1950]. The sheardiffusion model of Carter and Okubo [1965] was found to provide a good description of the development of cloud size with time. This model also accounts for the fact that the tracer distributions were not radially symmetric. Effective horizontal diffusivities lie between 0.02 and $0.3 \mathrm{~m}^{2} \mathrm{~s}^{-1}$. Reevaluation of published data from experiments in Lake Ontario [e.g., Murthy, 1976] and in the ocean [e.g., Okubo, 1971] supports both the applicability of the shear-diffusion model and the doubts raised against the appropriateness of the inertial subrange model for scales up to $1000 \mathrm{~m}$.
\end{abstract}

\section{Introduction}

The distribution of dissolved and suspended compounds in lakes and oceans is strongly influenced by the movement of the water. Water currents are turbulent in most situations. Therefore currents cannot be solely described by a set of deterministic equations. There always remains a stochastic component of motion superimposed on the average large-scale current pattern. Its adequate mathematical description has been, and still is, the aim of numerous theoretical and experimental investigations. Quantification of mixing processes is based on the understanding of the stochastic component of current patterns.

Progress has been different for the case of vertical and horizontal mixing, respectively. (Note that the terms vertical and horizontal are used instead of diapycnal and isopycnal.) Since in most situations vertical density gradients and/or the limited vertical extension of the water body keep vertical turbulent structures small, the local description of vertical turbulent fluxes by the first Fickian law is common practice for many, though not for all situations [Powell and Jassby, 1974; Wüest et al., 1996]. Various techniques have been developed to determine the coefficient of vertical turbulent diffusivity, e.g., the temperature and the tracer method [Powell and Jassby, 1974; Ledwell et al., 1993] as well as the method based on microstructure measurements of temperature or current velocity [Osborn and Cox, 1972; Oakey, 1982; Gregg, 1987]. In addition, there exist theoretical concepts to link vertical mixing with other hydrodynamic properties of the water column such as vertical stability and turbulent kinetic energy dissipation [Gargett, 1984] or internal wave shear [Gregg, 1989].

In contrast to the numerous field investigations on vertical turbulent mixing, research on horizontal turbulent mixing has been scarce. An explanation often invoked is that horizontal mixing is fast compared to vertical mixing so that the major concentration gradients develop along the vertical axis. While this is commonly true in small- to medium-sized lakes, large lakes and the ocean pose a different problem. In these water bodies, horizontal transport is dominated by the large-scale currents. Thus horizontal mixing is a consequence of both the fluctuations of the velocity field and the shear of the mean advective currents. This difficulty does rarely arise for vertical mixing, since vertical currents are usually absent and transport can be attributed to turbulence alone.

Recently, several researchers have used drifter experiments to study horizontal mixing [Kawai, 1985; Sanderson and Booth, 1991; Pal and Sanderson, 1992; Sanderson et al., 1995]. The statistics of drifter trajectories and its consequence on patch dispersion have found much attention [e.g., Sanderson and Booth, 1991, and references therein]. However, commonly, the number of drifters deployed is small (e.g., 5 to 10 as given by Sanderson et al. [1995]), and the calculation of average quantities by combining measurements from an ensemble of drifter experiments seems problematic.

Most experimental investigations on horizontal mixing were made with artificial tracers. For example, Okubo [1971], Kullenberg [1972], Ewart and Bendiner [1981], Yanagi et al. [1982], and Ward [1985] studied horizontal mixing in the ocean, and Murthy [1976] and Palmer et al. [1987] studied horizontal mixing in Lake Ontario. Quay et al. [1979] and Colman and Armstrong [1983] investigated horizontal mixing in very small lakes. 
dimensional distribution. If mixing is horizontally isotropic and the distribution radially symmetric, then $2 K_{\text {app }}=K_{m a}=K_{m i}$.

According to (4a) the apparent horizontal diffusivity $K_{\text {app }}$ can be determined from the actual rate of change of the cloud size $\sigma^{2}$. Since in several field investigations the cloud size was determined only once after the release of the tracer, $K_{\text {app }}$ is often approximated by the effective diffusivity $K_{\text {eff }}$ that is defined by replacing in (4a) the temporal derivative by a finite difference and by assuming $\sigma^{2}(t=0)=0$. Thus

$$
K_{\text {eff }} \cdot \sigma^{\iota} t
$$

The effective diffusivity is the temporal mean of the apparent diffusivity: $K_{\text {eff }}=t^{-1} \int_{0}^{t} K_{\text {app }}\left(t^{\prime}\right) d t^{\prime}$. In order to compare different published experiments, in the above equations, $\sigma^{2}$ is replaced by $\sigma_{r}^{2}$ whenever the specific data were evaluated in terms of $\sigma_{r}^{2}$ instead of $\sigma^{2}$.

Mixing by turbulence is a stochastic process. Theoretical models for horizontal mixing are based on the statistical properties of the small-scale turbulent velocity field and on its interaction with the large-scale velocity field. Thus in the strict sense of the statistical nature of mixing the models can only predict the mean temporal development of the concentration distribution for many identical experiments. The comparison of data with theoretical mixing models requires that the above quantities $\sigma_{m a}^{2}, \sigma_{m i}^{2}$, and $\sigma^{2}$ are evaluated for the ensemble mean concentration distribution determined from many identical experiments at identical diffusion times. Yet, the transient characteristics of most mixing situations do not allow us to conduct several mixing experiments that can be considered to be identical. Therefore as an approximation we interpret the temporal evolution of a concentration distribution of a single experiment within the framework of a specific mixing model.

In the next section, some published mixing models are described and their inherent assumptions analyzed. Next the experiments conducted during this research are presented. The final two sections are devoted to the interpretation of the results and to conclusions.

\section{Models for Horizontal Diffusion}

Theoretical diffusion models describe the temporal development of the statistical properties of a concentration distribution based on the properties of the velocity field that are assumed to be relevant for the mixing process. A turbulence model that has been used to describe tracer experiments in the ocean is the inertial subrange model [Kolmogorov, 1941] (application to dispersion by Batchelor [1950]; application to experiments by, e.g., Okubo [1971] and Ward [1985]). In this model the velocity field is assumed to be fully turbulent, isotropic, and stationary at scales of the cloud size. Eddies larger than the cloud size are supposed to cause advection, while smaller eddies are held responsible for diffusion relative to the center of mass of the concentration distribution. If the cloud size ranges within the scales of the inertial subrange of the turbulence spectrum, dimensional arguments can be used to predict the cloud size to increase with time $t$ as

$$
\boldsymbol{\sigma}^{2}=\text { const } \boldsymbol{\varepsilon} t^{3}
$$

where $\varepsilon$ is the dissipation rate of turbulent kinetic energy. In this model the velocity field is assumed to be three dimensional. Thus the statistical properties of the tracer distribution must be spherical symmetric; that is, the variance must have the same size in all directions. However, vertical density stratification and the limited vertical size of a well-mixed water body limit turbulence in the vertical. As shown by Monin and Ozmidov [1985], (5) remains valid for two-dimensional (horizontal) isotropic turbulence if the cloud size is within the inertial subrange of the turbulence spectrum. The horizontal diffusivity $K_{\text {app }}$ is calculated from (4a) and (5):

$$
K_{\text {app }}=\text { const } \varepsilon^{1 / 3} \sigma^{4 / 3}
$$

The mathematical form of the inertial subrange model (equations (5) and (6)) suggests using, as a more general approach, a power law with unknown exponent $m$ to describe the growth of $\sigma^{2}$ with time:

$$
\sigma^{2}=\text { const } t^{m}
$$

The corresponding horizontal diffusion coefficient is calculated from (4) and (7) and expressed as a function of the actual cloud size $\sigma$

$$
K_{\text {app }}(\sigma) \quad \text { const } \sigma^{2}
$$

Note that in all these models (except (4b)) the initial time $t=0$ does not necessarily correspond to the time when a tracer was introduced to the water body as an infinitesimally small cloud $\left(\sigma^{2}=0\right)$. If the initial tracer cloud has a finite size, $t=0$ represents the hypothetical time when the tracer cloud had zero size. In fact, a more general form of (7) would be $\sigma^{2}=$ const $\left(t+t_{0}\right)^{m}$ where $t_{0}$ is defined by the initial cloud size at $t=0: \sigma_{0}^{2}=$ const $t_{0}^{m}$.

Okubo [1971], on the basis of many different dye experiments in the surface layer of the ocean, compiled the most reliable data set. He combined the results from several experiments into an ensemble of measurements and subsequently compared it to model predictions. Deviations of the data of such an ensemble from a model curve (e.g., (7)) were then regarded as randomly distributed. The different environmental conditions prevailing during the different experiments were neglected. The application of the empirical model (7) to the entire data set suggested an exponent of 2.34, i.e., $\sigma_{r}^{2} \sim t^{2.34}$. Okubo suggested that for scales up to $1 \mathrm{~km}$, between $1 \mathrm{~km}$ and $10 \mathrm{~km}$, and above $10 \mathrm{~km}$ the data could be interpreted to follow $\sigma_{r}^{2} \sim t^{3}$ and thus support the inertial subrange model. He, however, stated that his interpretation based on the inertial subrange model "is by no means decisive" [Okubo, 1971]. As it turns out, for scales up to $1 \mathrm{~km}$ (for this range Okubo [1971] cited mainly data of Foxworthy et al. [1966]), individual clouds from a single experiment grow more slowly with time than predicted for the ensemble of experiments (see Figure 9b). This is also true for the experiments conducted by Murthy [1976] in the hypolimnion of Lake Ontario. All these experiments combined predict the distribution to increase according to $\sigma_{r}^{2} \sim t^{3.3}$, but individual experiments give exponents between 0.5 and 1.5 .

Joseph and Sendner [1958] developed a model for horizontal diffusion by postulating that the distance between an individual water parcel and the center of mass of the tracer distribution increases on average at a constant velocity. This leads to an exponent in (7) of $m=2$ and to an effective diffusivity $K_{\text {eff }}$ which increases linearly with time. Finally, we note that the Fickian law with constant diffusivity corresponds to $m=1$. Thus all these models combined cover a range for $m$ lying between 1 and 3 .

Yet, the most severe restriction of these models is their 
A detailed overview on horizontal mixing studies using tracer experiments is given by Peeters [1994].

In this work we describe experiments with uranin (an artificial fluorescent dye) that were conducted in several Swiss lakes. The largest of these lakes (Lake Neuchâtel) has a surface area of $218 \mathrm{~km}^{2}$, and the smallest (Lake Alpnach, a basin connected to Lake Lucerne) has a surface area of $4.8 \mathrm{~km}^{2}$. The experiments were carried out far from the lake boundaries where the isopycnals are horizontal, except for temporal distortions due to internal waves. In the following discussion, horizontal mixing is thus considered to be equivalent to isopycnal mixing.

When comparing experiments on horizontal mixing, problems arise not only due to the different physical and orographic conditions in these lakes but also because different investigators evaluate their results with different concepts. In this work, mixing is defined by those effects of the horizontal velocity field which cause the concentration distribution of a passive tracer cloud to spread relative to its center of mass (relative diffusion). The movement of the center of mass is considered to be due to (large scale) advection. To avoid confusion, the above definition of mixing should be carefully distinguished from other concepts which also can be found in the literature. Consider, e.g., the fate of a chemical which is introduced into the water body by some local source. Advection is responsible for the (fast) transport of the chemical away from the source to other locations in the lake. The time to reach the far end of the lake is often called "mixing time." Note that this time depends on the large-scale current pattern which contributes only little to the dilution of the tracer cloud by surrounding water. It is by dilution that the concentration within the moving cloud decreases. Mixing as employed in this study includes all effects causing the tracer cloud to spread, i.e., the small-scale turbulent motions which cause dilution of the tracer cloud and the interaction between this small-scale motion with the largescale current pattern.

To quantify the term "mixing" as defined above, a measure for the spread of a concentration distribution is required. The distribution can be characterized by quantities such as the total mass $M_{O}$, the coordinates of the centre of mass $x_{i_{s}}$, and variance and covariance $\sigma_{\boldsymbol{x}: \boldsymbol{x}}$ :

$$
\begin{aligned}
& M_{o}=\int \quad c(x, y, z) d x d y d z \\
& \left.x_{i=}=\overline{M_{C}} \iiint \quad y, z\right) d x d y d z \\
& \left.\sigma_{\lambda}=\frac{1}{M_{4}} \iint \quad x_{\imath} \quad x_{i_{s}}\right)\left(x_{j} \quad x_{i_{s}}\right) c(x, y, z) d x d y d z
\end{aligned}
$$

where $i, j=1,2,3, x_{i}$ are the Cartesian coordinates $\left(x_{1}=\right.$ $x, x_{2}=y$, and $\left.x_{3}=z\right)$ and $c(x, y, z)$ is the spatial concentration field at a given time. Since mixing neither influences the total mass nor the movement of the centre of mass, mixing is solely described by the temporal change of the variances and covariances $\sigma_{x_{i} x_{j}}$. Moments of higher order than 2 are not considered here. Thus the complex information of a general three-dimensional concentration distribution is reduced to that contained in a three-dimensional normal distribution.

To analyze horizontal mixing, the concentration distribution is integrated vertically. This leaves us with a two-dimensional distribution that generally is not isotropic. The eigenvalues of the matrix

$$
\Sigma_{2}=\left(\begin{array}{ll}
\sigma_{:} & \sigma_{x y} \\
\sigma & \sigma_{w}
\end{array}\right)
$$

yield the variances in the direction of the major and minor principal axes ( $\sigma_{m a}^{2}$ and $\sigma_{m i}^{2}$ ) of the vertically integrated distribution. The directions of the principal axes are determined by the eigenvectors of $\Sigma_{2}$. The instantaneous rate of mixing can be characterized by turbulent diffusion coefficients $K_{m a}$ and $K_{m i}$ that are related to the temporal evolution of $\sigma_{m a}^{2}$ and $\sigma_{m i}^{2}$ :

$$
d \sigma_{m a}^{2} / d t=2 K_{m a} \quad d \sigma_{m i}^{2} / d t=2 K_{m i} .
$$

If $K_{m a}$ and $K_{m i}$ are constant, (2) corresponds to the results of the first Fickian law for the spreading of a distribution far from the boundaries. However, as shown below the variances rarely grow linearly with time. Therefore the diffusion coefficients defined by (2) are time (or scale) dependent.

The horizontal extension of the vertically integrated concentration distribution (thereafter referred to as cloud size) can be defined as [Okubo, 1971]

$$
\sigma^{2}=2 \sigma_{m a} \sigma_{m i}
$$

Note that $\sigma^{2}$, in contrast to, e.g., the total variance, $\sigma_{m a}^{2}+$ $\sigma_{m i}^{2}$, does not change under the influence of laminar flow, even if velocity gradients distort the shape of a tracer patch. Consider, as an example, a circular tracer cloud influenced by laminar currents along the $x$ axis with velocity gradient in the $y$ direction. The currents transform the circle into an ellipse. When time passes, the major axis of the ellipse will grow and turn into the $x$ direction, while the minor axis will shrink. Thus $\sigma_{m a}$ increases and $\sigma_{m i}$ decreases with time, while $\sigma^{2}$ remains constant.

In the case of a two-dimensional normal distribution the lines of equal concentration are ellipses. The ellipse that corresponds to $e^{-1}(37 \%)$ of the maximum concentration encloses $63 \%$ of the total mass of the distribution and has an area of $F=\pi \dot{\sigma}^{2}$. The ellipse with major and minor axis of length $3 \sigma_{m a}$ and $3 \sigma_{m i}$, respectively, encloses $99 \%$ of the total mass of the distribution.

In many publications on horizontal mixing, symmetric distributions were constructed from measured concentrations [e.g., Okubo, 1971; Murthy, 1976]. For different concentration values $c$ the areas enclosed by iso-concentration lines were determined. The equivalent radius $R(c)$ is then defined as the circle with an area equal to the area enclosed by the isoconcentration line. Instead of using $\sigma^{2}$ the size of the cloud is then defined by the variance $\sigma_{r}^{2}$ of the inverse function $c(R)$. In the case of a two-dimensional normal distribution the isoconcentration line which represents $e^{-1}$ of the maximum concentration encloses an area of $F=\pi \sigma_{r}^{2}$ and thus $\sigma_{r}^{2}$ and $\sigma^{2}$ are equal.

In addition to cloud size $\sigma^{2}$ and variance $\sigma_{r}^{2}$, which both are measures of the extension of a tracer cloud, a scale of diffusion defined as $l_{r}=3 \sigma_{r}$ is commonly in use [e.g., Okubo, 1971].

In analogy to (2) the temporal evolution of $\sigma^{2}$ serves to define an apparent horizontal diffusion coefficient $K_{\text {app }}$ by the relation

$$
d \sigma^{2} / d t=4 K_{\mathrm{app}}
$$

The factor 4 on the right-hand side reflects the fact that in contrast to (2), (4a) describes the evolution of a two- 
radial symmetry. This is in contradiction with most experimental results. Non-radially symmetric distributions can be described by shear-diffusion models [Carter and Okubo, 1965; Okubo, 1967, 1968; Kullenberg, 1972; Young et al., 1982]. For the development of these models one assumes that the velocity field can be separated into two parts, one including the turbulent fluctuations and the other corresponding to the (nonturbulent) large-scale advective flow. The diffusion time (elapsed time since tracer release) is supposed to be sufficiently large so that the autocorrelation of the velocity fluctuations becomes negligible. Consequently, the spread of a tracer distribution caused by the turbulent part of the velocity field can be modeled using the Fickian law [Taylor, 1921]. Assuming the turbulent diffusion coefficients $K_{x}, K_{y}$, and $K_{z}$ to be spatially constant, the advection-diffusion equation can be written as

$\frac{\partial c}{\partial t}+u \frac{\partial c}{\partial x}+v \frac{\partial c}{\partial y}+w \frac{\partial c}{\partial z}=K_{x} \frac{\partial^{2} c}{\partial x^{2}}+K_{y} \frac{\partial^{2} c}{\partial y^{2}}+K_{z} \frac{\partial^{2} c}{\partial z^{2}}$

where $u, v$, and $w$ are the components of the nonturbulent part of the velocity field in the $x, y$, and $z$ direction, respectively. If the mean velocity field is assumed to be purely horizontal and to lie along the $x$ axis with $u=-y a-z b$, where the horizontal and vertical shear, $a$ and $b$, respectively, are constant, one obtains

$$
\frac{\partial c}{\partial t}-(a y+b z) \frac{\partial c}{\partial x}=K_{x} \frac{\partial^{2} c}{\partial x^{2}}+K_{y} \frac{\partial^{2} c}{\partial y^{2}}+K_{z} \frac{\partial^{2} c}{\partial z^{2}} .
$$

This corresponds to the model by Carter and Okubo [1965], except for a constant advection term in the $x$ direction.

The variances and covariances of the concentration distribution obeying the Carter and Okubo [1965] model are given by

$$
\begin{aligned}
& \sigma_{x x}=2 K_{x} t+\frac{2}{3}\left(a^{2} K_{y}+b^{2} K_{z}\right) t^{3} \\
& \sigma_{y y}=2 K_{y} t \\
& \sigma_{z z}=2 K_{z} t \\
& \sigma_{x y}=-K_{y} a t^{2} \\
& \sigma_{x z}=-K_{z} b t^{2} \\
& \sigma_{y z}=0 .
\end{aligned}
$$

These results were obtained by introducing a momentum generating function (this method is demonstrated by Okubo [1967]) and assuming the initial tracer distribution to be pointlike. The solution for $c(x, y, z, t)$, found by Carter and Okubo [1965] (see also Okubo [1968]) is in agreement with the variances and covariances given in (11). However, our (11) disagrees with the expressions given by $O k u b o$ [1968] for $\sigma_{x_{i} x_{j}}$. Okubo's expressions must be incorrect; for example, his equation for $\sigma_{x x}$ is not symmetric in the $z$ and $y$ coordinates although the velocity field is.

From (11) the variance in the direction of the minor and major principal axes and the cloud size can be calculated. Assuming $K_{x}=K_{y} \equiv K_{h}$, one obtains

$$
\begin{aligned}
\sigma_{m a}^{2}= & 2 K_{h} t+\frac{1}{3}\left(a^{2} K_{h}+b^{2} K_{z}\right) t^{3} \\
& +\sqrt{K_{h}^{2} a^{2} t^{4}+\left[\frac{1}{3}\left(a^{2} K_{h}+b^{2} K_{z}\right) t^{3}\right]^{2}} ; \\
\sigma_{m i}^{2}= & 2 K_{h} t+\frac{1}{3}\left(a^{2} K_{h}+b^{2} K_{z}\right) t^{3} \\
& -\sqrt{K_{h}^{2} a^{2} t^{4}+\left[\frac{1}{3}\left(a^{2} K_{h}+b^{2} K_{z}\right) t^{3}\right]^{2}} ; \\
\sigma^{2}= & 2 \sigma_{m a} \sigma_{m i}=2 \sqrt{4 K_{h}^{2} t^{2}+\frac{1}{3} K_{h}\left(a^{2} K_{h}+4 b^{2} K_{z}\right) t^{4}} .
\end{aligned}
$$

If the velocity shear is zero $(a=b=0)$, one obtains $\sigma^{2}=4 K_{h} t$. This is the solution to (4a) for a constant diffusivity $K_{h}$ (Fickian diffusion) and zero initial variance. For large times the cloud size $\sigma^{2}$ increases as $t^{2}$ like in the Joseph and Sendner [1958] model. However, the latter describes radially symmetric distributions, in contrast to the Carter and Okubo [1965] model for which $\sigma_{m a}^{2}$ and $\sigma_{m i}^{2}$ are usually different. In fact, for long diffusion times the Carter and Okubo model predicts that $\sigma_{m a}^{2} \sim t^{3}$ and $\sigma_{m i}^{2} \sim t$ (see (12)). The major principal axis of the tracer distribution then turns into the direction of the current $\left(\sigma_{\mathrm{xy}} / \sigma_{\mathrm{xx}} \underset{t \rightarrow \infty}{\rightarrow} 0\right)$.

If the tracer distribution at $t=0$ is not point-like but a three-dimensional normal distribution with initial variance $\sigma_{x x_{o}}, \sigma_{y y_{o}}$ and $\sigma_{z z_{o}}$, one obtains:

$$
\begin{aligned}
\sigma_{x x}=2 K_{x} t+\frac{2}{3}\left(a^{2} K_{y}+b^{2} K_{z}\right) t^{3}+\sigma_{x x o}+\left(a^{2} \sigma_{y y o}+b^{2} \sigma_{z z o}\right) t^{2} \\
\sigma_{y y}=2 K_{y} t+\sigma_{y y o} \\
\sigma_{z z}=2 K_{z} t+\sigma_{z z o} \\
\sigma_{x y}=-K_{y} a t^{2}-a \sigma_{y y o} t \\
\sigma_{x z}=-K_{z} b t^{2} \quad b \sigma_{z z o} t \\
\sigma_{y z}=0
\end{aligned}
$$

From these results, $\sigma_{m a}^{2}, \sigma_{m i}^{2}$, and $\sigma^{2}$ can be calculated as above.

Other shear-diffusion models differ from the model by Carter and Okubo [1965] with respect to their nonturbulent part of the velocity field. Okubo [1967] extended the Carter and Okubo model by including oscillating currents. Kullenberg [1972] considered horizontally rotating currents with current speed decreasing with depth. In these models the cloud size still increases with time but superimposed there is an oscillatory variation. For instance, for large times the Kullenberg model causes the cloud size to increase as $\sigma^{2} \sim t^{2}\left[\frac{10}{3}+\frac{2}{3}\right.$ $\cos (2 \omega t)]^{1 / 2}$, where $\omega$ is the angular frequency of the rotating current. Expressions of $\sigma_{x_{i} x_{j}}$ for the Kullenberg model are given by Peeters [1994].

The above models were generalized by Young et al. [1982], who investigated the interaction between turbulent diffusion and the velocity field produced by internal waves. They found that for large diffusion times the spread of a concentration distribution is dominated by the velocity shear of a steady current compared to the oscillating components and that $\sigma_{x x} \sim t^{3}$ as in the model by Carter and Okubo [1965].

Sanderson and Okubo [1988] analyzed the influence of a random field of internal waves by perturbation theory on the Navier-Stokes equation. They assumed the spectrum of the horizontal velocity to be proportional to the square of the frequency and found, as for the inertial subrange model, that $\sigma^{2} \sim t^{3}$ for cloud sizes between $10^{2}$ and $10^{4} \mathrm{~m}^{2}$. Yet for cloud sizes larger than $10^{4} \mathrm{~m}^{2}$ the exponent of the time dependence drops below 3 .

In connection with the interpretation of drifter experiments, recent research relates the fractal properties of drifter trajectories to particle dispersion [e.g., Sanderson and Booth, 1991]. Sanderson and Booth find that particle dispersion can be modeled as accelerated fractional Brownian motion providing $\mathrm{Oku}$ $b o$ 's [1971] $\sigma^{2} \sim t^{2.34}$ power law. Note that, especially for small scales of diffusion, drifter and tracer experiments are not 
Table 1a. General Information on the Eight Tracer Release Experiments

\begin{tabular}{|c|c|c|c|c|c|c|c|c|}
\hline Experiment $^{\mathrm{a}}$ & Date & $\begin{array}{c}\text { Surface Area } \\
\text { of Basin, } \\
\mathrm{km}^{2}\end{array}$ & $\begin{array}{l}\text { Maximum Depth } \\
\text { of Basin, } m\end{array}$ & $\begin{array}{c}\text { Duration of } \\
\text { Experiment, } \\
\text { hours }\end{array}$ & $\begin{array}{l}\text { Number of } \\
\text { Surveys }^{\mathrm{b}}\end{array}$ & $\begin{array}{l}\text { Tracer Mass } \\
\text { Released, g }\end{array}$ & $\begin{array}{l}\text { Depth of } \\
\text { Tracer Release, } \\
\text { m }\end{array}$ & $\begin{array}{c}\text { Stability at Depth } \\
\text { of Tracer } \\
\text { Release, } \mathrm{s}^{-2}\end{array}$ \\
\hline & Oct. 8, 1990 & 4.8 & 35 & 73 & 7 & 1000 & & $2 \times 10^{-4}$ \\
\hline & March 25, 1991 & 22.0 & 151 & 52 & $6(7)$ & 2000 & & $7 \times 10^{-6}$ \\
\hline & June 24,1991 & 217.9 & 154 & 48 & 4 & 1500 & & $4 \times 10^{-4}$ \\
\hline & Sept. 19, 1991 & 22.0 & 200 & 49 & $4(5)$ & 1000 & & $8 \times 10^{-4}$ \\
\hline & Sept. 30, 1991 & 22.0 & 200 & 12 & 3 & 250 & & $3 \times 10^{-4}$ \\
\hline & Oct. 10, 1991 & 22.0 & 200 & 9 & $2(3)$ & 200 & & $4 \times 10^{-4}$ \\
\hline & June 10, 1992 & 22.0 & 151 & 50 & 7 & 305 & & $1 \times 10^{-4}$ \\
\hline & Oct. 6,1992 & 22.0 & 151 & 52 & 8 & 265 & & $3 \times 10^{-4}$ \\
\hline
\end{tabular}

${ }^{\mathrm{a}}$ The names of the experiments stand for Alpnachersee (Alp), Vitznauersee (Vitz), Urnersee (Urn), and Lac de Neuchâtel (Neu). The first three basins are part of Lake Lucerne.

'Numbers in parentheses are total number of surveys including those with unsufficient tracer mass recovery. The latter were not considered in the following chapters.

directly comparable since they are sensitive to different ranges of the velocity spectrum.

The following discussion will mainly focus on two models, the inertial subrange model [Batchelor, 1950] and the sheardiffusion model by Carter and Okubo [1965]. The former received wide attention through the work of Okubo [1971], while the latter provides better agreement with most measurements due to its anisotropy. The shear-diffusion models with oscillating currents are not considered any further, since most field data do not allow us to properly determine the various parameters of these models.

\section{Experiment}

A total of eight tracer experiments were conducted at different times of the year in the upper hypolimnion of several Swiss lakes. Table 1a summarizes information on these lakes as well as on the experiments. Alpnachersee (Alp), Vitznauersee (Vitz), and Urnersee (Urn) are all basins of Lake Lucerne located in the center of Switzerland (for a general description of the basins, see Aeschbach-Hertig [1994]). Lac Neuchâtel (Neu), located in the western part of Switzerland, is one of the largest Swiss lakes.

The fluorescent dye sodium fluorescein (uranin, $\mathrm{C}_{20} \mathrm{H}_{10} \mathrm{Na}_{2} \mathrm{O}_{5}$ ) was used as artificial tracer in all experiments. This dye does not significantly adsorb on suspended particles and is nontoxic. However, it is decomposed by photolysis and strongly affected by hydrolysis at a $\mathrm{pH}<7$ [Smart and Laidlaw, 1977]. All experiments took place in carbonate lakes ( $\mathrm{pH}$ well above 7) at depths of about $20 \mathrm{~m}$ where the light intensity is less than $2 \%$ of the intensity at the lake surface. Thus, during the experiments of a few days, decomposition of uranin was negligible.

The tracer was released through a tube of $1.5-\mathrm{m}$ length containing 40 holes of $2.5-\mathrm{mm}$ diameter. Groups of five holes around the tube were located about $20 \mathrm{~cm}$ apart. The tube was connected to a hose and placed vertically into the water at the target depth. Between 200 and $2000 \mathrm{~g}$ of uranin (see Table 1a) was dissolved in $70 \mathrm{~L}$ of warm surface water on board the ship and pumped through the holes into the lake within about $60 \mathrm{~s}$. By this procedure the tracer was diluted with at least $100 \mathrm{~m}^{3}$ of water immediately after release. The specific expansion coefficient of water due to uranin, $\rho^{-1} \partial \rho / \partial C$, was measured to be approximately $4.4 \times 10^{-4} \mathrm{~m}^{3} \mathrm{~kg}^{-1}$. Except for Vitz 1 , the vertical stability frequency of the water column at the depth of tracer release, $N^{2}=-g \rho^{-1} d \rho / d z$, was $10^{-4} \mathrm{~s}^{-2}$ or greater
(Table 1a). Thus, even under the most conservative assumption (dilution of $2 \mathrm{~kg}$ of uranin into $100 \mathrm{~m}^{3}$ of water), the uranin/water mixture sank by less than $1 \mathrm{~m}$ after release (except for Vitz 1).

The dye concentration was measured in situ at a rate of 10 $\mathrm{Hz}$ using a fluorometer (either "Variosens II," Impulsphysik GmbH, or "Backscat," Geological and Marine Instrumentation). The output signal was passed through an isolation amplifier and subsequently made available to the computer by analogue-digital conversion using a data acquisition board (National Instruments NB-MIO-16L25). A conductivitytemperature-depth (CTD) probe (Multisonde MS 040, Meerestechnik Elektronik $\mathrm{GmbH}$ ) attached to the fluorometer provided information on pressure (resolution $0.01 \mathrm{dbar}$ ), temperature (resolution $0.001^{\circ} \mathrm{C}$ ), and electrical conductivity (resolution $0.1 \mu \mathrm{S} \mathrm{cm}^{-1}$ ) at a rate of $8 \mathrm{~Hz}$. Depth and water density were calculated from these data. The exact position of the ship was obtained from a microwave positioning system (Trisponder Systems, Del Norte Technology Inc.) at a rate of $1 \mathrm{~Hz}$ (resolution $\pm 1 \mathrm{~m}$ ). The data flow from the different instruments was coordinated by a data acquisition program developed for a Macintosh computer. On-line display of pressure and tracer concentration enabled an efficient survey of the tracer distributions.

During each experiment the tracer cloud was repeatedly mapped, each mapping requiring a few hours. Each mapping will be called a survey. The number of surveys for each experiment is listed in Table 1a. Examples of tracer clouds constructed from individual surveys from different experiments are shown in Figure 1. During the surveys the fluorometer and the CTD probe were moved up and down in a yo-yo-like manner. Vertical direction was changed whenever the probes were above or below the tracer cloud. Usually the ship was steered along straight lines through the tracer cloud at a constant speed of about $1 \mathrm{~m} \mathrm{~s}^{-1}$ and turned outside the cloud. The position of the probes was calculated from the position of the ship by using a velocity-dependent horizontal drag distance between ship and probe.

Every up or down cast is called a vertical profile. Each survey typically consists of more than 100 vertical profiles. The distance between vertical profiles along the ship track is typically less than $15 \mathrm{~m}$, i.e., less than the length of the dashes shown in Figure 1. Vertical integration of the concentration in each profile provides a two-dimensional concentration distribution $c(x, y)$. In addition, horizontal integration allowed calculation 


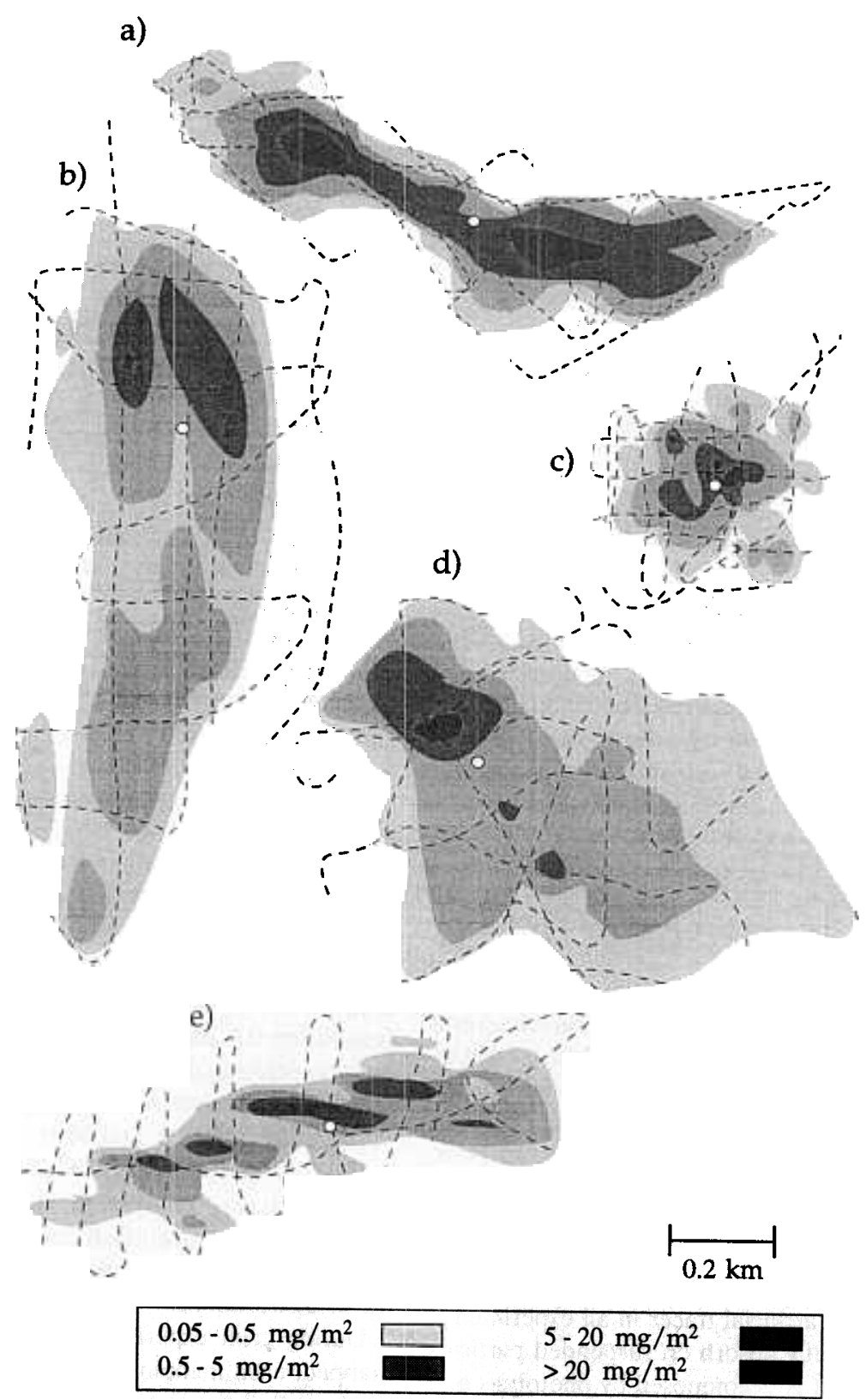

Figure 1. Examples of concentration distributions constructed from surveys of different experiments: (a) Vitznauersee (Vitz 1), 30 hours after release; (b) Urnersee (Urn 1), 25 hours; (c) Vitz 3, 13 hours, (d) Lac de Neuchâtel (Neu), 11 hours; (e) Alpnachersee (Alp), 28 hours. The dashed lines show the ship track during the surveys. The open circles mark the center of mass of the dye distribution. Note that the total amount of released tracer varies between experiments (see Table $1 b$ ).

for each survey of the total observed tracer mass. In most experiments the tracer mass recovered was on average more than $50 \%$ of the released mass (Table $1 \mathrm{~b}$ ).

During most of the experiments, drifters were released at the same depth as the tracer. From the position of the drifters before and after a survey an average linear drift velocity was determined. The coordinates of the individual profiles were corrected for the average drift in proportion to the time difference between the start of the survey and the time when the particular profile was taken. As it turned out, this correction is small and does not significantly change the tracer distribution. Two-dimensional integration of the linearly interpolated distribution $c(x, y)$ provides the total tracer mass, the coordinates of the center of mass, the variances in the directions of the principal axes, and the cloud size of the tracer distributions.

The initial patch size of the tracer immediately after tracer release was estimated by assuming the concentration to be at a constant value inside and to be zero outside a region having a radius of about $10 \mathrm{~m}$ in the horizontal and a total height of approximately $h \approx 1.5 \mathrm{~m}$ in the vertical (only in the experi- 
Table 1b. Characteristic Data of the Eight Tracer Release Experiments

\begin{tabular}{|c|c|c|c|c|c|c|}
\hline Experiment & $\begin{array}{l}\text { Estimated Cloud } \\
\text { Size at } t=0 \\
\qquad \mathrm{~m}^{2}\end{array}$ & $\begin{array}{c}\text { Tracer Mass } \\
\text { Recovered }^{\mathrm{a}} \\
\text { (Min. - Max.), } \%^{\text {Min. }}\end{array}$ & $\begin{array}{c}\text { Tracer Mass } \\
\text { Recovered, } \\
\text { (Average), } \\
\%\end{array}$ & $\begin{array}{c}\sigma_{m a} / \sigma_{m i} \\
\text { (Min. - Max.) }\end{array}$ & $\begin{array}{l}\sigma_{m a} / \sigma_{m i} \\
\text { (Average) }\end{array}$ & $\begin{array}{l}\text { Average Velocity } \\
\text { of Center of Mass, } \\
\qquad \mathrm{cm} \mathrm{s}^{-1}\end{array}$ \\
\hline Alp & 300 & $14-51$ & $33 \pm 5$ & $1.4-9.9$ & $2.8 \pm 1.1$ & $0.7 \pm 0.1$ \\
\hline Vitz 1 & 160 & (19) $44-200$ & $110 \pm 20$ & $1.8-6.7$ & $3.3 \pm 0.7$ & $1.8 \pm 0.3$ \\
\hline $\mathrm{Neu}$ & 200 & $31-51$ & $40 \pm 5$ & $1.8-2.4$ & $2.2 \pm 0.2$ & $1.8 \pm 0.3$ \\
\hline Urn 1 & 50 & (9) $40-76$ & $60 \pm 10$ & $1.8-3.1$ & $2.5 \pm 0.3$ & $0.9 \pm 0.1$ \\
\hline Urn 2 & 50 & $54-122$ & $90 \pm 20$ & $1.5-4.8$ & $2.8 \pm 1.0$ & $2.6 \pm 0.7$ \\
\hline Un 3 & 90 & (13) $78-99$ & $90 \pm 10$ & $2.8-5.6$ & $4.2 \pm 1.4$ & $2.9 \pm 0.5$ \\
\hline Vitz 2 & 50 & $73-188$ & $130 \pm 20$ & $1.1-7.9$ & $3.6 \pm 1.0$ & $1.1 \pm 0.2$ \\
\hline Vitz 3 & 50 & $39-153$ & $110 \pm 20$ & $1.1-4.5$ & $2.5 \pm 0.5$ & $0.9 \pm 0.2$ \\
\hline
\end{tabular}

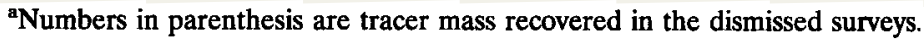

ment Vitz $1 h \approx 7 \mathrm{~m}$ ). A drift of the ship during tracer release causes an elongation of the tracer distribution in the drift direction. The resulting increase in the initial size of the tracer distribution can be estimated from the monitored ship movement during tracer release. Initial cloud sizes were calculated according to (3) to range between 50 and $300 \mathrm{~m}^{2}$ (Table $1 \mathrm{~b}$ ). The estimated initial height $h$ was compared to the concentration distribution of several profiles taken immediately after tracer release. The height $h$ estimated from the profiles was $h \leq 1.5 \mathrm{~m}$ in all experiments except Vitz 1 where $h \approx 7 \mathrm{~m}$. The initial vertical variance of the tracer cloud is given by $\sigma_{z z_{O}}=\frac{1}{12} h^{2}$, which is approximately $\sigma_{z z_{O}} \approx 0.2 \mathrm{~m}^{2}$ for all experiments except for Vitz 1 where $\sigma_{z z_{0}} \approx 4.4 \mathrm{~m}^{2}$.

\section{Results}

As an example, Figure 2a shows the temporal development of the tracer cloud in the experiment Vitz 1 , represented by the ellipses with principle axes of length $3 \sigma_{m a}$ and $3 \sigma_{m i}$, respectively. Orientation and size of the principle axes were calculated from the matrix $\Sigma_{2}$ (see (1)). For a two-dimensional normal distribution the area of the ellipses is equal to $4.5 \pi$ times the cloud size (see (3)). The ellipses would then represent the iso-concentration line corresponding to about $1 \%$ of the maximal concentration and enclose $99 \%$ of the total tracer mass. Cloud 6 represents the tracer distribution shown in Figure 1a. Cloud 7 was measured about 2 days after the tracer release and marks the end of this particular experiment.

The linear velocity of the center of mass was calculated for each time interval between two consecutive surveys. In the experiment Vitz 1 these velocities reach a maximum value of $2.7 \mathrm{~cm} \mathrm{~s}^{-1}\left(2.3 \mathrm{~km} \mathrm{day}^{-1}\right)$. For each experiment the average velocity was calculated as the mean of the interval velocities (Table 1b). They range between 0.8 and $2.9 \mathrm{~cm} \mathrm{~s}^{-1}(0.6$ and $2.5 \mathrm{~km}$ day $^{-1}$ ). Direction and magnitude of these velocities can strongly vary within short periods of time. For example, in the tracer experiments Urn 2 and Urn 3 which were conducted on 2 consecutive days, the direction of the advective current changed from southwest to northeast.

Most tracer distributions are elongated rather than radially symmetric (Figures 1 and 2a), in spite of the initial circular shape of the cloud in some of the experiments (Vitz 2, Vitz 3, Urn 1, Urn 2). The variance along the major principal axis $\sigma_{m a}^{2}$ mostly grows faster than the variance along the minor principal axis $\sigma_{m i}^{2}$ (Figures $2 b$ and $2 c$ ), although it should be noted that the spatial orientation of these axes is not fixed. For each experiment the temporal evolution of $\sigma_{m a}^{2}$ and $\sigma_{m i}^{2}$ is de- scribed by power laws of the form $\sigma_{m a}^{2} \sim t^{p}$ and $\sigma_{m i}^{2} \sim t^{q}$. Averaging the exponents obtained for all experiments yields the following mean values: $p=1.5 \pm 0.2$ for $\sigma_{m a}^{2}$ and $q=1.0 \pm 0.1$ for $\sigma_{m i}^{2}$. Thus the ratio $\sigma_{m a}^{2} / \sigma_{m i}^{2}$ increases with time. Cloud size and variance in the direction of the principal axes observed during the experiments Vitz 1 and Vitz 2 are shown in Figures $2 \mathrm{~b}$ and $2 \mathrm{c}$.

The temporal increase of the cloud size $\sigma^{2}$, calculated according to (3), is shown in Figure 3 for all experiments. Except for a constant factor, the functional dependence of the cloud size on time is rather equal. The same functional dependence is found for $\sigma_{r}^{2}$, the variance of a cloud assumed to be radially symmetric. However, the $\sigma_{r}^{2}$ values are 2-3 times smaller than $\sigma^{2}$. Remember that for two-dimensional normal distributions, the two values, $\sigma^{2}$ and $\sigma_{r}^{2}$, should be equal. Obviously, the clouds have more complex shapes. For instance, the center of mass of the tracer distributions does not usually coincide with the location of maximum concentration as required for a normal distribution (see Figure 1). In addition, the development of "islands" with enhanced concentration was often observed. These islands and the shift of the center of mass relative to the location of maximum concentration result in variances of the tracer distribution that are larger than the variances of the constructed radially symmetric distribution. Since the variances $\sigma_{r}^{2}$ range between 100 and $10^{5} \mathrm{~m}^{2}$, our experiments cover scales of diffusion between 30 and $1000 \mathrm{~m}$.

In order to test different models for horizontal mixing, the errors of the derived cloud characteristics (cloud size $\sigma^{2}$, variance in the direction of the principle axes $\sigma_{m a}$ and $\sigma_{m i}$ ) have to be known. They were estimated by a procedure that is similar to the bootstrap estimator. For each survey a hypothetical concentration distribution was generated by constructing a hypothetical ship track. Concentrations along the hypothetical track were determined by interpolation from the measured tracer concentrations. The distance between the hypothetical profiles was chosen to give the same spatial resolution as the real profiles of the survey. The variances $\sigma_{m a, H}^{2}$ and $\sigma_{m i, H}^{2}$ and the cloud size $\sigma_{H}^{2}$ of the hypothetical concentration distribution were calculated and compared to the values obtained for the measured distribution.

The difference between the two sets of variances gives a semiquantitative measure for the uncertainty originating from the reconstruction of a continuous tracer distribution from discrete data points with limited spatial resolution. However, this is only true provided that the subgrid concentration variance is smaller than the variance measured for the grid points. 

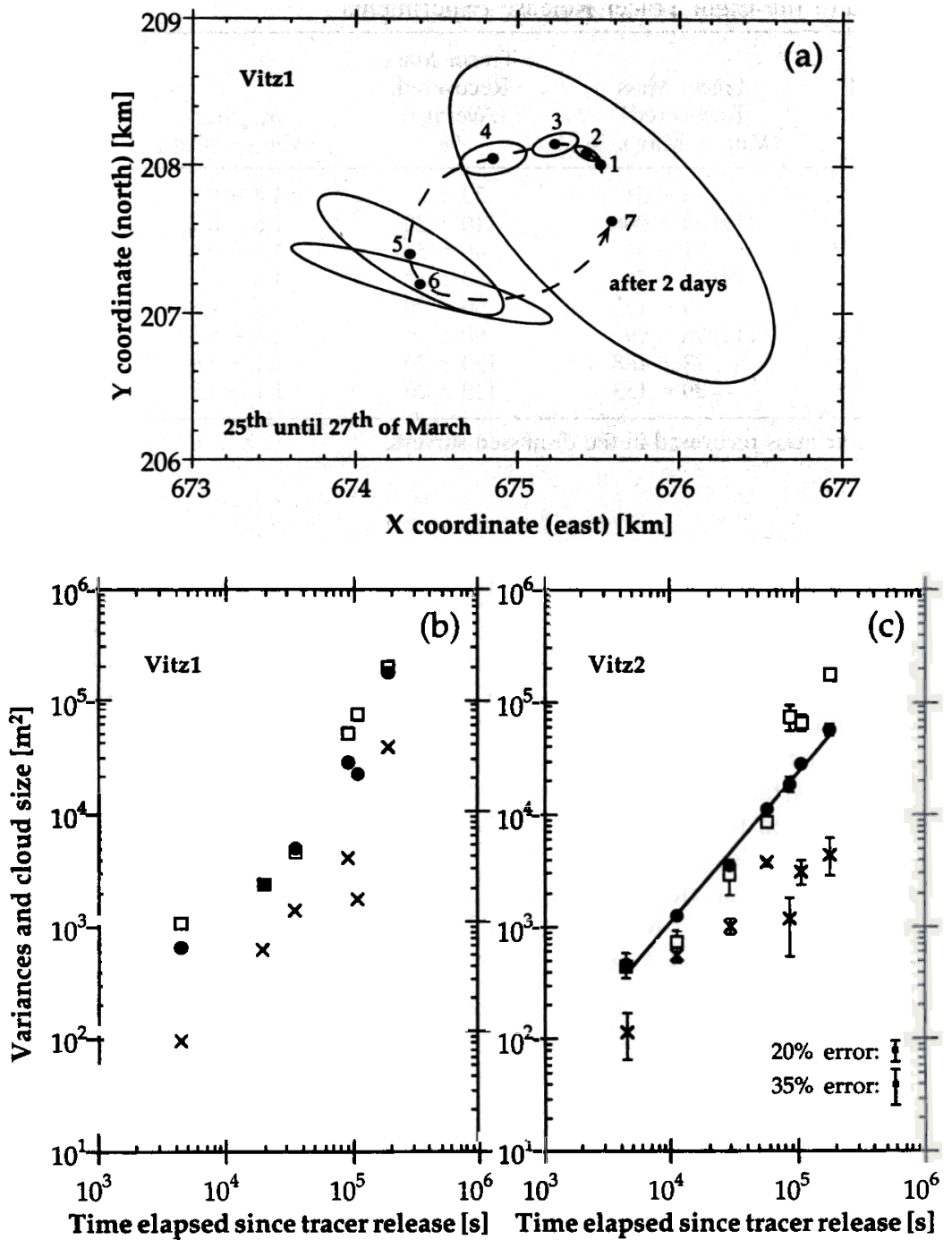

Figure 2. Temporal development of tracer distributions in Vitznau basin. (a) Schematic representation of the changing tracer cloud during the experiment Vitz 1 . Each cloud is represented by an ellipse with principle axes of length $3 \sigma_{m a}$ and $3 \sigma_{m i}$ calculated from the matrix $\Sigma_{2}$ (see (1)). The solid circles are the center of mass. Ellipse 6 corresponds to the tracer cloud shown in Figure 1a. (b) Temporal development of cloud size $\sigma^{2}=2 \sigma_{m a} \sigma_{m i}$ (solid circles) and variance in the direction of the major (open squares) and minor (crosses) principal axes for experiment Vitz 1. (c) As in Figure 2b, for Vitz 2. Fitting the cloud size with a power function $\sigma_{m}^{2}=A_{1} t^{A_{2}}$ (solid line) yields the exponent $A_{2}=1.4$.

This assumption seems to be reasonable given the mostly smooth shape of the tracer clouds and the fact that the errors turn out to be rather similar for the various surveys. Typically, the error (defined as 1 standard deviation) of the cloud size $\sigma^{2}$ is $20 \%$, and the errors of the variances $\sigma_{m a}^{2}$ and $\sigma_{m i}^{2}$ are 25 and $35 \%$, respectively (see, e.g., Figure $2 \mathrm{c}$ ). The errors can be slightly larger for those surveys which were carried out very shortly after the release of the tracer.

From the cloud size as function of time the horizontal diffusion coefficient can be determined either from (4a) or (4b). Our experiments, conducted in the upper hypolimnion of lakes of medium size, yield $K_{\text {eff }}$ values between 0.02 and $0.3 \mathrm{~m}^{2} \mathrm{~s}^{-1}$ in agreement with measurements in the hypolimnion of Lake Ontario [Murthy, 1976]. Typical $K_{\text {eff }}$ values in the ocean are larger by a factor of 3-5 [e.g., Okubo, 1971], while in very small ice-covered lakes, $K_{\text {eff }}$ can be much smaller than in our experiments [e.g., Colman and Armstrong, 1983].
During the observational period of each experiment (3-4 days) the vertical variance of the tracer distributions, calculated by averaging tracer concentrations at constant density for all vertical profiles of a survey, increased only very slowly. The average concentration profile as a function of density was then transferred to a concentration profile as a function of depth using the mean density profile determined for the particular survey. Except for the experiments Vitz 1 and Urn 3 the precision of the measurements was sufficient to rule out that the vertical variance increases by more than $0.2 \mathrm{~m}^{2}$ day $^{-1}$. Thus the vertical diffusion coefficient, $K_{z}=(1 / 2)\left(d \sigma^{2} / d t\right)$, must be of the order of $1 \times 10^{-6} \mathrm{~m}^{2} \mathrm{~s}^{-1}$ or less. Diapycnal diffusion coefficients in lakes that are determined from water temperature or dissolved chemical substances are usually larger by a factor of 3 or more [e.g., Li, 1973; Imboden and Wüest, 1995]. However, these tracers are usually spread across the whole lake area. Recent investigations in the ocean [Ledwell and 
Watson, 1991] and in lakes [Wüest et al., 1996] suggest that vertical mixing occurs mainly at the bottom boundary of the water basin followed by horizontal mixing of the vertically mixed components. Since during the above experiments the tracer did usually not hit the boundaries, the small $K_{z}$ value can be interpreted as reflecting the vertical diffusivity in the "interior" of the water body.

\section{Discussion}

In this section the experimental data are compared with the different diffusion models described in section 3. A $\chi^{2}$ test is employed to check whether the cloud size predicted by a specific model, $\sigma_{\text {mod }}^{2}\left(t_{i} ; A_{1}, \cdots, A_{p}\right)$, is compatible with the experimental data. The parameters $A_{1}$ to $A_{p}$ are adjusted by a weighted least squares fit. The quantity

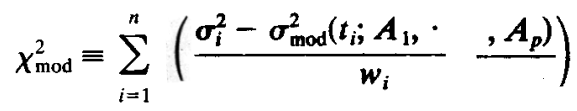

is calculated, where $w_{i}$ is the absolute error of $\sigma_{i}^{2}$ and $i$ denotes the $i$ th survey of an individual experiment. The $w_{i}$ were calculated by assuming that the relative error of the cloud size is $20 \%$. The variable $\chi^{2}$ is supposed to be $\chi^{2}$ distributed with $k$ degrees of freedom, where $k$ is the difference between the number of data points (surveys) and the number of fitting parameters. If the probability of the variable $\chi^{2}$ to be larger than or equal to $\chi_{\text {mod }}^{2} P\left(\chi^{2} \geq \chi_{\text {mod }}^{2}\right)$, is less than $1 \%$, then the model $\sigma_{\text {mod }}^{2}\left(t_{i} ; A_{1}, \cdots, A_{p}\right)$ is rejected.

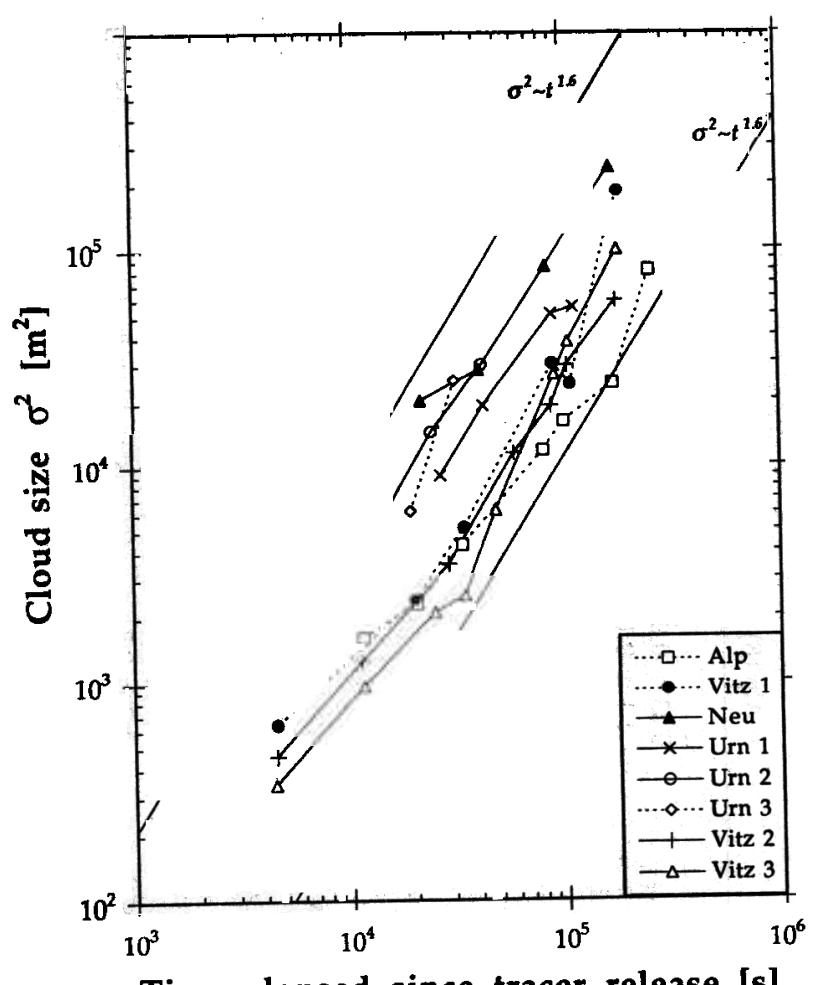

Time elapsed since tracer release [s]

Figure 3. Temporal increase of cloud size $\sigma^{2}$ of all experiments calculated according to (3).

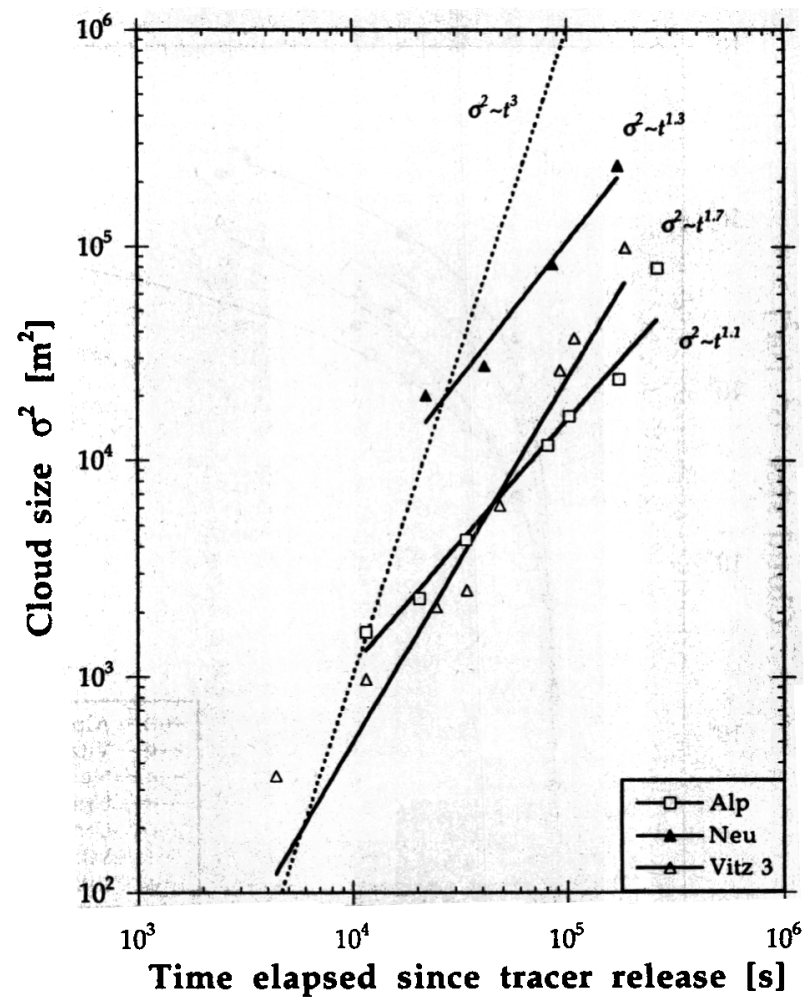

Figure 4. Fitting of individual experiments to the empirical model $\sigma_{m}^{2}=A_{1} t^{A_{2}}$. The relative error was assumed to be equal for all measurements. A growth of the cloud size as $t^{3}$ is indicated by the dashed line.

\subsection{Inertial Subrange Model}

According to (5) the inertial subrange model implies that the cloud size grows with time as $t^{3}$. Fitting the cloud sizes to the power law $\sigma_{\text {mod }}^{2}=A_{1} t^{A_{2}}$ yields exponents $A_{2}$ between 1.1 and 1.7 for the different experiments. As an example, three fits are shown in Figure 4. They clearly deviate from the $t^{3}$ curve. The fitting parameters for all experiments are listed in Table 2 . The mean exponent calculated for those experiments consisting of at least four surveys is $1.4 \pm 0.1$. According to the $\chi^{2}$ test the probability $P\left(\chi^{2} \geq \chi_{\text {mod }}^{2}\right)$ for the model function $\sigma_{\text {mod }}^{2}=A_{1} t^{3}$ is less than $1 \%$, even if the error of the measured cloud sizes is raised to $50 \%$. Thus the model $\sigma_{\bmod }^{2}=$ const $t^{3}$ must be rejected.

As pointed out by Csanady [1973], for short diffusion times a finite size of the initial cloud $\sigma_{O}^{2}$ may simulate an almost linear increase of the cloud size with time. In order to test the potential influence of the initial cloud size on the above result, the model is extended by a third fitting parameter: $\sigma_{\text {mod }}^{2}=$ $A_{1}\left(t+\left|A_{3}\right|\right)^{A_{2}}$. The time $t=-\left|A_{3}\right|$ can be interpreted as the hypothetical release time when the cloud size was zero. The modified model function was fitted to all experiments for which at least three surveys exist (Figure 5). This includes all experiments except Urn 3. The estimated initial cloud sizes (Table 1b) were included in the data sets with a relative error of $100 \%$. Now the exponents $A_{2}$ range between 1.3 and 2.3 with a mean value of $1.6 \pm 0.1$ (see Table 2). This is not compatible with an increase of $\sigma^{2}$ with elapsed time raised to the power of 3 . The inertial subrange model also can be extended to account for the finite size of the initial cloud: $\sigma_{\text {mod }}^{2}=A_{1}\left(t+\left|A_{2}\right|\right)^{3}$. The $\chi^{2}$ test of this model yields the probability $P\left(\chi^{2} \geq \chi_{\text {mod }}^{2}\right)$ less 


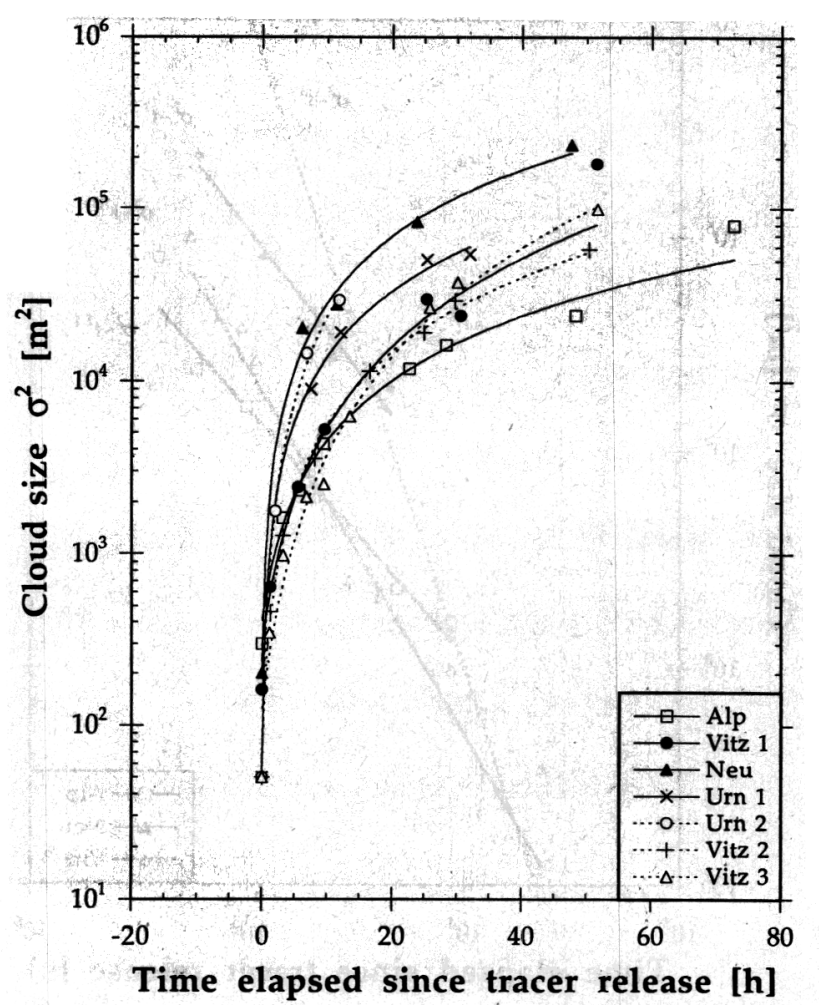

Figure 5. Cloud size as function of elapsed time since tracer release modeled by $\sigma_{m}^{2}=A_{1}\left(t+\left|A_{3}\right|\right)^{A_{2}}$. The initial cloud size at $t=0, \sigma_{O}^{2}$, is included into the fit with a relative error of $100 \%$. The errors for the other cloud sizes are $20 \%$.

than 2\% for Alp and Vitz 3 and less than 1\% for the other experiments. Thus, the modified inertial subrange model also has to be rejected as a meaningful interpretation of the data set.

\subsection{The Shear Model of Carter and Okubo [1965]}

Shear diffusion models allow the description of non-radially symmetric tracer distributions. The model by Carter and Okubo [1965] yields the following function for the cloud size (see (12)):

$$
\sigma_{\text {miod }}^{2}
$$

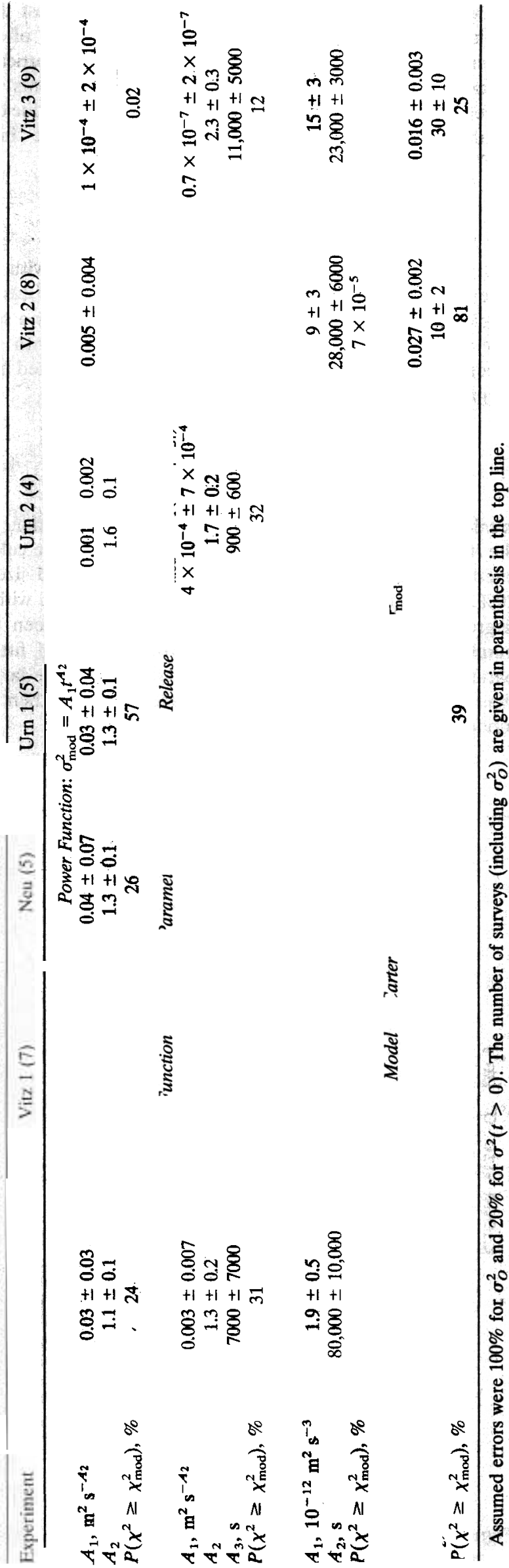




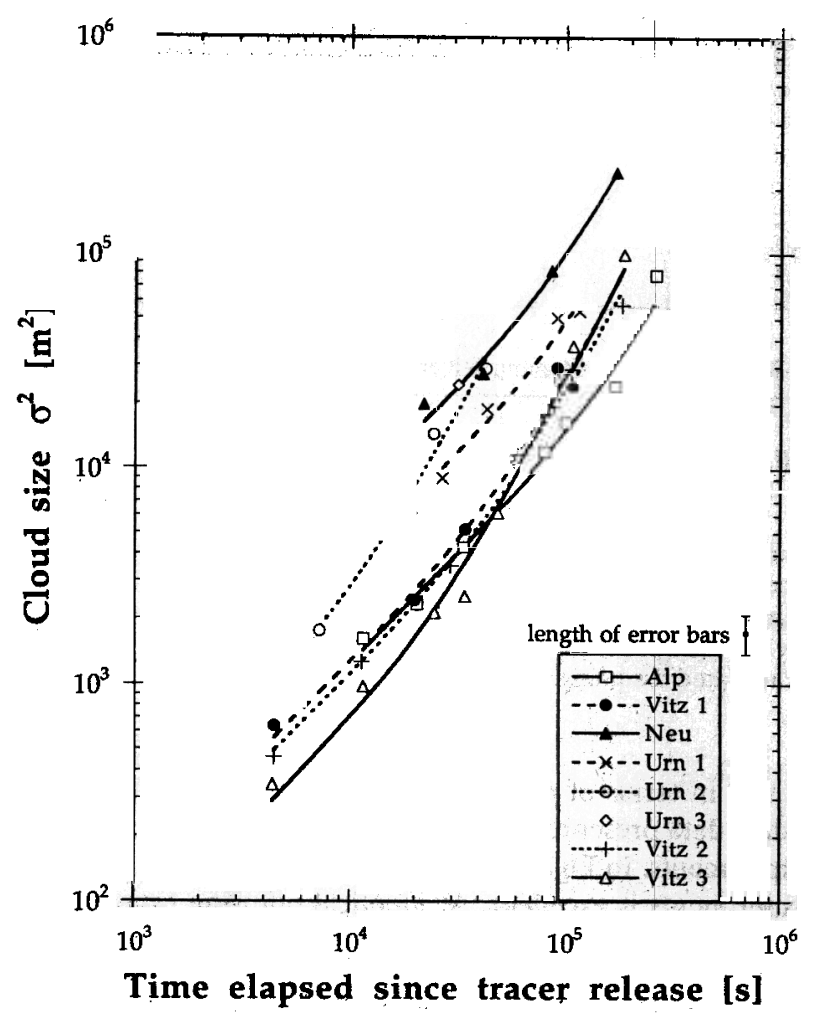

Figure 6. Fit of measured cloud sizes of individual experiments to the shear-diffusion model by Carter and Okubo [1965] using the function $\sigma_{m}^{2}=2\left(4 A_{1}^{2} t^{2}+\frac{1}{3} A_{1} A_{2} t^{4}\right)^{1 / 2}$. All data have the same relative error of $20 \%$ as shown by the error bar on the right side of the figure.

where $A_{1}=K_{h}$ and $A_{2}=K_{h}(\partial u / \partial y)^{2}+4 K_{z}(\partial u / \partial z)^{2}$. The dependence of the cloud size on time should therefore gradually change from $\sigma^{2} \sim t$ to $\sigma^{2} \sim t^{2}$. From the tests performed for the power law models (7) it is to be expected that this model is in fair agreement with the field data. Indeed, fitting of the individual experiments (i.e., all experiments with at least three surveys) provides an excellent description of the development of the cloud size with time (Figure 6). The probability $P\left(\chi^{2} \geq \chi_{\text {mod }}^{2}\right)$ is about $5 \%$ for Vitz 1 and ranges between 25 and $80 \%$ for all other experiments (Table 2). Thus the model (15) is in accordance with all tested experiments. This is true whether the initial cloud size $\sigma_{O}^{2}$ is zero or assumes the value listed in Table $1 \mathrm{~b}$. For Vitz 1 , the only experiment with a somewhat larger initial vertical variance $\left(\sigma_{z z_{0}} \approx\right.$ $\left.4.4 \mathrm{~m}^{2}\right)$, the influence of $\sigma_{z z_{O}}$ on the spread of the tracer cloud is discussed at the end of this section.

The parameters $A_{1}$ and $A_{2}$ and the values of $\chi_{\text {mod }}^{2}$ are listed in Table 2. Since according to the shear diffusion model the fitted parameters are linked to observable quantities (velocity shear), it has to be checked whether the results are plausible and consistent with the velocity shear that was observed during the tracer experiments. For the experiments conducted in Lake Lucerne (all except Neu), the average value for $A_{1}$, which in the model corresponds to the horizontal diffusivity $K_{h}$, is $0.04 \mathrm{~m}^{2} \mathrm{~s}^{-1}$ with variation between 0.016 and $0.09 \mathrm{~m}^{2} \mathrm{~s}^{-1}$. Note that these values are smaller than the effective diffusivities calculated above since the former represent real turbulent exchange while the latter additionally describe the interaction of the turbulence with the velocity shear. Horizontal turbulent diffusivity in Lac Neuchâtel (Neu) is significantly larger than in Lake Lucerne $\left(0.18 \mathrm{~m}^{2} \mathrm{~s}^{-1}\right)$. This lake is larger and exposed to stronger winds.

The fitting values of the second parameter, $A_{2}$, vary between $1 \times 10^{-11}$ and $400 \times 10^{-11} \mathrm{~m}^{2} \mathrm{~s}^{-3}$. According to the shear diffusion model, $A_{2}$ depends on the square of the velocity shear in both horizontal and vertical direction. This may explain its great variability even between experiments conducted in the same lake basin (compare Urn 1 and Urn 2 in Table 2). Note that the shear diffusion model of Carter and Okubo [1965] assumes the shear to be constant in space in time. In nearsurface flows of the sea, however, velocity shear appears to be scale dependent [Kawai, 1985]. In addition, the spectrum of horizontal velocity has no spectral gap, and thus a clear distinction between a purely turbulent and a nonturbulent part of the velocity field is impossible. However, although detailed (space and time dependent) measurements of the velocity shear are not available for our measurements, we want at least to check whether the order of magnitude of $A_{2}$ is reasonable. We choose $K_{z}=1 \times 10^{-6} \mathrm{~m}^{2} \mathrm{~s}^{-1}$, as suggested by the observed vertical spread of the tracer during the experiments, and $K_{h}=4 \times 10^{-2} \mathrm{~m}^{2} \mathrm{~s}^{-1}$, as determined from $A_{1}$. Drifters and moored current meters employed during the tracer experiments provided values for the vertical shear, $(\partial u / \partial z)$, in the range of $1 \times 10^{-3}$ to $5 \times 10^{-3} \mathrm{~s}^{-1}$. For the estimation of the horizontal shear it is assumed, guided by Figure $2 a$, that the typical horizontal current velocity of $2 \mathrm{~cm} \mathrm{~s}^{-1}$ decays over a distance of $1 \mathrm{~km}$. This yields $\partial u / \partial y=2 \times 10^{-5} \mathrm{~s}^{-1}$. With these values $A_{2}=K_{h}(\partial u / \partial y)^{2}+4 K_{z}(\partial u / \partial z)^{2}$ should lie between $10^{-11}$ and $10^{-10} \mathrm{~m}^{2} \mathrm{~s}^{-3}$, in accordance with the range of values determined from the experiments.

The Carter and Okubo [1965] model describes not only the cloud size but also the variance in the direction of the major and minor principal axes as function of time (see (12)). The corresponding model functions contain three fitting parameters $A_{i}$ :

$$
\begin{aligned}
\sigma_{\text {mi mod }}^{2} & =2 A_{1} t+1 / 3\left(A_{1} A_{2}+A_{3}\right) t^{3} \\
+ & \sqrt{A_{1}^{2} A_{2}^{2} t^{4}+\left[1 / 3\left(A_{1} A_{2}^{2}+A_{3}\right) t^{3}\right]^{2}} \\
\sigma_{\text {mi,mod }}^{2}= & 2 A_{1} t+1 / 3\left(A_{1} A_{2}^{2}+A_{3}\right) t^{3} \\
& \sqrt{A_{1}^{2} A_{2}^{2} t^{4}+\left[1 / 3\left(A_{1} A_{2}^{2}+A_{3}\right) t^{3}\right]^{2}}
\end{aligned}
$$

where $A_{1}=K_{h}, A_{2}=\partial u / \partial x$, and $A_{3}=K_{z}(\partial u / \partial z)^{2}$. Note that these equations imply a point-like initial concentration distribution. The parameters are determined to minimize the sum

$$
\begin{aligned}
& \gamma_{\text {mod }}^{2}=\sum_{i=1}^{n}\left(\frac{\sigma_{m i, i}^{2}}{\sigma_{m a, \bmod }^{2}} \frac{\left(t_{i}, A_{1}, A_{2}, A_{3}\right.}{w_{m a, i}}\right. \\
& +\sum^{n}\left(\frac{\sigma_{m, i, i}^{2}}{\sigma_{m i, \bmod }^{2}\left(t_{i}, A\right.} \frac{\left.A_{2}, A_{3}\right)}{w_{m i, i}}\right)
\end{aligned}
$$

where $w_{m a, i}$ and $w_{m i, i}$ are the absolute errors (1 standard deviation) of $\sigma_{m a, i}^{2}$ and $\sigma_{m i, i}^{2}$, respectively. Only experiments with at least four surveys (yielding eight independent data points, i.e., four values of $\sigma_{m a}^{2}$ and $\sigma_{m i}^{2}$ each) were considered for the fitting procedure (Table 3 ). The agreement between model and data is good (Figure 7, solid lines and solid circles and triangles). From the fit parameters the cloud size was 
Table 3. Fit Parameters for the Shear-Diffusion Model by Carter and Okubo [1965]

\begin{tabular}{|c|c|c|c|c|c|c|c|}
\hline & Alp & & itz 1 & $\mathrm{Neu}$ & Urn & Vitz 2 & Vitz 3 \\
\hline \multirow[t]{2}{*}{$\begin{array}{l}\text { No. of surveys } \\
A_{1}, \mathrm{~m}^{2} \mathrm{~s}^{-1} \\
A_{2}, 10^{-5} \mathrm{~s}^{-1} \\
A_{3}, 10^{-11} \mathrm{~m}^{2} \mathrm{~s}^{-3} \\
P\left(\chi^{2} \geq \chi_{\text {mod }}^{2}, \%\right. \\
\end{array}$} & \multicolumn{2}{|c|}{$\begin{array}{c}14 \\
0.012 \pm 0.006 \\
2.3 \pm 0.7 \\
-0.006 \pm 0.6 \\
1 \times 10^{-4}\end{array}$} & $\begin{array}{c}12 \\
0.018 \pm 0.005 \\
4.5 \pm 1.5 \\
3 \pm 3 \\
0.4 \\
\end{array}$ & $\begin{array}{c}8 \\
0.13 \pm 0.05 \\
1.7 \pm 1.2 \\
4.8 \pm 8.5 \\
7.5 \\
\end{array}$ & $\begin{array}{c}8 \\
0.07 \pm 0.02 \\
2.8 \pm 0.6 \\
3 \pm 4 \\
31\end{array}$ & $\begin{array}{c}14 \\
0.027 \pm 0.002 \\
4.3 \pm 0.7 \\
1 \pm 1 \\
11\end{array}$ & $\begin{array}{c}16 \\
0.014 \pm 0.004 \\
0.9 \pm 7 \\
4 \pm 3 \\
5.5\end{array}$ \\
\hline & $\begin{array}{c}\text { Lake Ontario } \\
\text { ED1 } \\
\end{array}$ & $\begin{array}{c}\text { Lake Ontario } \\
\text { ED5 }\end{array}$ & $\begin{array}{c}\text { Lake Ontario } \\
\text { ED3 }\end{array}$ & Mikawa Bay & Banana River & $\begin{array}{c}\text { off Southern } \\
\text { California (e) }\end{array}$ & $\begin{array}{l}\text { Pacific (1000-m } \\
\text { depth) }\end{array}$ \\
\hline $\begin{array}{l}\text { No. of surveys } \\
A_{1}, \mathrm{~m}^{2} \mathrm{~s}^{-1} \\
A_{2}, 10^{-11} \mathrm{~m}^{2} \mathrm{~s}^{-3} \\
P\left(\chi^{2} \geq \chi_{\text {mod }}^{2}\right), \%\end{array}$ & $\begin{array}{c}4 \\
0.05 \pm 0.01 \\
4 \pm 3 \\
22\end{array}$ & $\begin{array}{c}4 \\
0.16 \pm 0.05 \\
10 \pm 10 \\
3\end{array}$ & $\begin{array}{c}3 \\
0.02 \pm 0.02 \\
0.06 \pm 3 \\
0.3\end{array}$ & $\begin{array}{c}11 \\
0.066 \pm 0.003 \\
2900 \pm 500 \\
99\end{array}$ & $\begin{array}{c}5 \\
0.09 \pm 0.05 \\
2 \pm 10 \\
0.007\end{array}$ & $\begin{array}{c}3 \\
0.11 \stackrel{ \pm 0.05}{7000 \pm 4000} \frac{8}{8}\end{array}$ & $\begin{array}{c}3 \\
0.04 \pm 0.02 \\
-0.01 \pm 1 \\
2.5\end{array}$ \\
\hline
\end{tabular}

The top part of this table shows application of the model to the variances in the direction of the principal axes (see (16).) The values for $\sigma_{m a}^{2}$ and $\sigma_{m i}^{2}$ were assumed to have an error of $35 \%$. The bottom part of this table shows application of the model (15) to published data for the cloud size $\sigma^{2}$ or $\sigma_{r}^{2}$ : Lake Ontario [Murthy, 1976], Mikawa Bay [Yanagi et al., 1982], Banana River and “off Southern California (e)" [Okubo, 1971 ], and in the Pacific [Ewart and Bendiner, 1981]. The value of $\chi^{2}$ was calculated assuming an error of $20 \%$ for all data.

calculated and compared to the measured size (Figure 7, dashed line and circles with dot inside). Note that minimizing (17) does not exactly yield the same parameter values as (15). However, the parameter values obtained from the fits are similar in both cases.

Figure 7 demonstrates that the model of Carter and Okubo [1965] is suitable for the description of the cloud size as well as of the variances in the direction of the principal axes. According to the $\chi^{2}$ test the Carter and Okubo model is consistent with the data from the experiments Vitz 2 and 3, Neu, and Urn 1 (Table 3). Using an error of about 35\% for the variance in the direction of both principal axis, the probability $P\left(\chi^{2} \geq \chi_{\text {mod }}^{2}\right)$ is small for the experiments Alp and Vitz 1 (Table 3). One possible explanation may be the rather large and elongated initial concentration in these particular experiments.

In order to explore the influence of the initial cloud size, the data from Vitz 1 were tested by the modified Carter and Okubo [1965] model (13). The initial cloud size is assumed to be a three-dimensional normal distribution with initial variance of $210 \mathrm{~m}^{2}, 30 \mathrm{~m}^{2}$, and $4.4 \mathrm{~m}^{2}$ for the major principle, the minor principal, and the vertical axis, respectively. The agreement between measurement and model is good (Figure 8). However, statistics do not significantly improve since the errors of the initial values are large and a fourth fitting parameter is required.

\subsection{Application of the Carter and Okubo [1965] Model to Published Data}

The above evaluation clearly demonstrates that for scales up to $1000 \mathrm{~m}$, horizontal transport can be better described by a combination of velocity shear and (small scale) turbulent diffusion than by the inertial subrange turbulence model. One might argue that this conclusion is biased due to the small size of the lakes in which the experiments were conducted. Since the size of the lake limits the scale of turbulence, the cloud might outgrow the inertial subrange scale and thus invalidate the conditions for the model.

In order to test this possible objection, several published experiments which were conducted in large lakes (e.g., in Lake Ontario [Murthy, 1976] or in the ocean [Okubo, 1971]) and which cover diffusion scales up to $1000 \mathrm{~m}$ are compared with our own results (Figure 9). Some authors present their data in terms of the radially symmetric cloud size $\sigma_{r}^{2}$ (Figure 9a) and others in terms of $\sigma^{2}$ as defined in (3) (Figure 9b). The published data presented in Figure $9 \mathrm{~b}$ exclusively stem from ocean experiments. In Figure 9a the agreement between the different experiments is excellent. At identical diffusion times the vari-
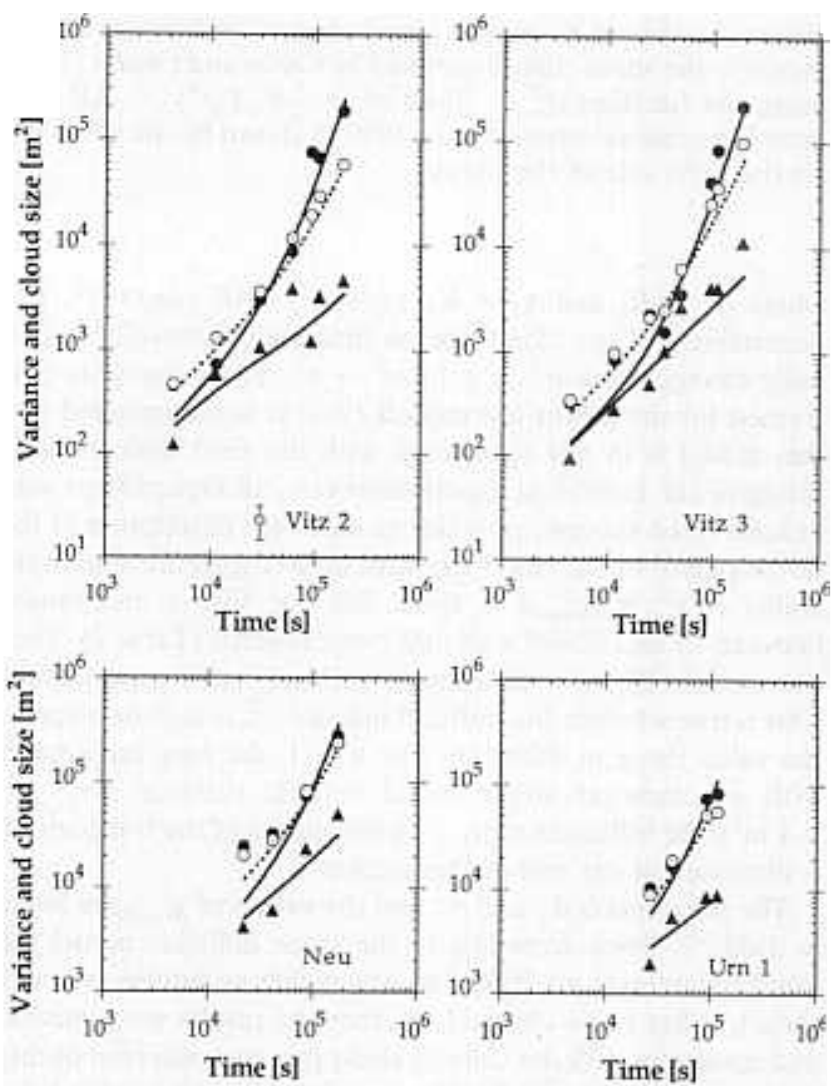

Figure 7. Application of the shear-diffusion model to the measured variance along the major (solid circles) and minor (solid triangles) principal axes from four experiments. The fit (solid lines) was carried out simultancous for $\sigma_{m a}^{2}$ and $\sigma_{m i}^{2}$ by assuming a relative error of $35 \%$ to all measurements (see error bar in top left panel for Vitz 2). Cloud size was calculated from the fit parameters (dashed lines) and compared to the measurements (circles with dot inside). 
ance $\sigma_{r}^{2}$ measured in the hypolimnion of Lake Ontario are approximately the same as in our experiments. In the ocean the clouds are generally larger than in our lakes. This was to be expected since most of the results for the ocean stem from experiments in the surface mixed layer [Okubo, 1971; Yanagi et al., 1982]. However, for diffusion scales up to $1000 \mathrm{~m}$ the exponents of the power law model in individual experiments are similar for all the published and for our experiments, independent of whether they were conducted in the surface layer of the ocean or in the epilimnion or hypolimnion of a lake. In fact, the power law model yields exponents of $m=0.5$ to 1.5 for the hypolimnion and of $m=1.6$ to 2.2 for the epilimnion of Lake Ontario (data of Murthy [1976] and of Palmer et al. [1987]). For the ocean experiments by Yanagi et al. [1982] and those compiled by $O k u b o$ [1971], exponents range between 1 and 1.9 for the diffusion scales up to $1000 \mathrm{~m}$ discussed here (for this range Okubo's data mostly stem from Foxworthy et al. [1966] cited by Okubo [1971]).

Many authors state that the tracer distributions observed were usually elongated [e.g., Okubo, 1971; Murthy, 1976]. Therefore we have reevaluated some of the published data for scales up to $1000 \mathrm{~m}$ with the model by Carter and Okubo [1965]. Since errors of $\sigma^{2}$ or $\sigma_{r}^{2}$ are usually not available, it is not possible to formally calculate the probability for rejection of the various models. However, the results shown in Figure 10 demonstrate that the shear-diffusion model would provide a better description of these experiments than the inertial subrange model. The $\chi_{\text {mod }}^{2}$ given in Table 3 were calculated by assuming that the relative errors of $\sigma^{2}$ and $\sigma_{r}^{2}$ are $20 \%$.

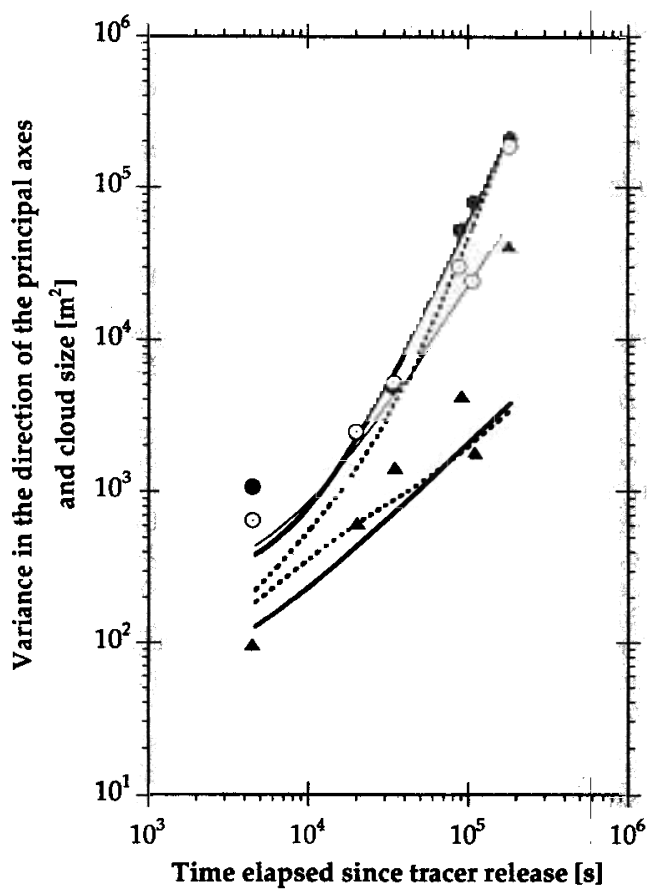

Figure 8. Comparison of fits to Vitz 1 applying the sheardiffusion model with (bold lines) and without (bold dashed lines) inclusion of the initial variances. The solid circles represent the variance along the major principle axis, $\sigma_{m a}^{2}$; the solid triangles represent the variance along the minor principle axis, $\sigma_{m i}^{2}$; and the circles with the dot inside represent the cloud size $\sigma^{2}$. The influence of the initial variance on the calculated cloud size (thin solid line) is negligible.

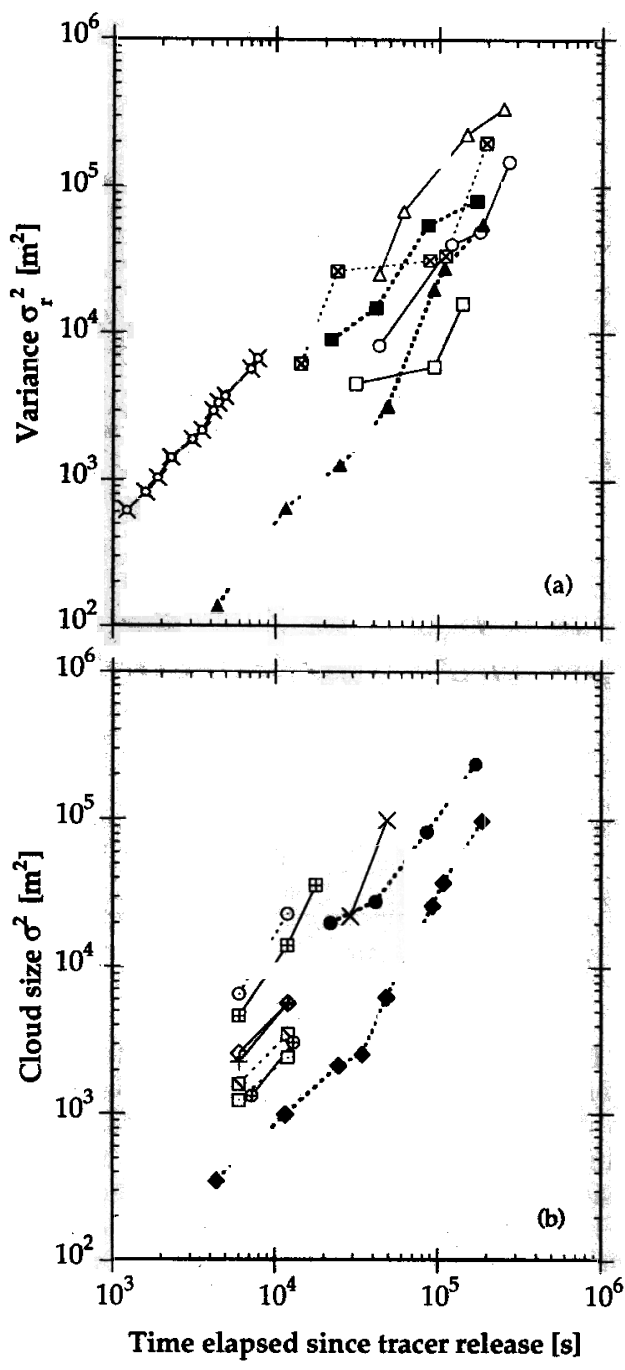

Figure 9. Comparison of our results with experiments conducted in Lake Ontario and in the ocean. (a) Radially symmetric variance $\sigma_{r}^{2}$ for the experiments Neu (solid squares) and Vitz 3 (solid triangles) and for different experiments in Lake Ontario (open squares, triangles, and circles, Murthy [1976]) and in the ocean (squares with cross inside, Okubo [1971]; crosses with circle in center, Yanagi et al. [1982]). (b) Cloud size $\sigma^{2}$ for the experiments Neu (solid circles) and Vitz 3 (solid diamonds) and for individual experiments in the ocean (crosses and circles with plus inside, Kullenberg [1972]; squares with plus inside, squares with dot inside, squares with slash inside, plusses, circles with dot inside, and open diamonds, Okubo [1971]). Lines connect measurements of individual experiments.

\section{Conclusions}

The main results of our investigation are that in none of the experiments did the cloud size grow as $t^{3}$. Furthermore, the growing clouds always became elongated and not radially symmetric. According to the $\chi^{2}$ test the inertial subrange model has to be rejected for almost all of our experiments independent of whether the initial cloud size of the tracer distribution is assumed to be finite or zero. The inertial subrange model reached probabilities $P\left(\chi^{2} \geq \chi_{\text {mod }}^{2}\right)$ larger than $1 \%$ only for the experiments Alp and Vitz 3, but even in these cases, $P\left(\chi^{2} \geq \chi_{\text {mod }}^{2}\right)$ remained below $2 \%$. In contrast, the shear diffusion model of 


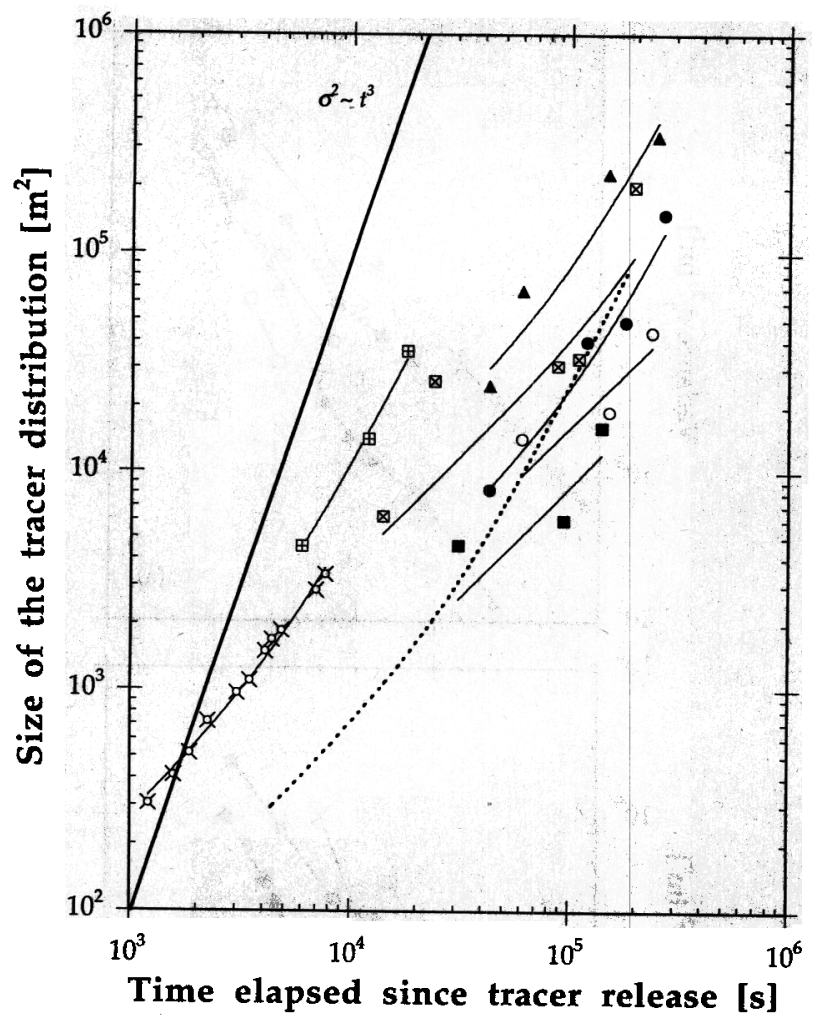

Figure 10. Application of the shear-diffusion model by Carter and Okubo [1965] to data from experiments in Lake Ontario (solid triangles, circles, and squares, Murthy [1976]) and in the ocean (squares with cross inside and squares with plus inside, Banana River and "off Southern California," Okubo [1971]; crosses with circle in center, Yanagi et al. [1982]; open circles, Ewart and Bendiner [1981]). Only experiments with at least three surveys were considered. Since some of the authors evaluated their data in terms of the variance $\sigma_{r}^{2}$, it was assumed that the latter is equal to the cloud size $\sigma^{2}$. For comparison the dashed line shows the model for the experiment Vitz 3.

Carter and Okubo [1965] provides a good description of the temporal development of cloud size as well as of the elongated cloud shape. According to the $\chi^{2}$ test the model description of the cloud size is consistent with all experiments. Although this does not prove the shear diffusion model to be the only model to describe the horizontal spread of tracer distributions, it clearly shows that the shear diffusion model is compatible with the data and cannot be rejected.

Effective horizontal diffusion coefficients lie between 0.02 and $0.3 \mathrm{~m}^{2} \mathrm{~s}^{-1}$. If the effect of the velocity shear is removed, as is the case in the shear diffusion model, the remaining horizontal (turbulent) diffusivity is between 0.02 and $0.18 \mathrm{~m}^{2} \mathrm{~s}^{-1}$. Assuming vertical diffusion to follow Fick's law, the vertical spread of the tracer distributions can be described with a diffusivity of about $1 \times 10^{-6} \mathrm{~m}^{2} \mathrm{~s}^{-1}$ or less. This value tends to be smaller than those determined from water temperature or natural tracers. The difference between these diffusivities can be understood since the former value is only affected by the interior mixing, whereas the latter includes boundary mixing which commonly is considered to cause a large vertical transport.

A new analysis of several published diffusion experiments shows that the development of individual cloud sizes for scales up to $1000 \mathrm{~m}$ can be described by the shear diffusion model.
The relation $\sigma^{2} \sim t^{3}$ suggested by, e.g., Okubo [1971] and Murthy [1976], appears, at least for scales up to $1000 \mathrm{~m}$, to be a consequence of combining measurements from different experiments into one data set and assuming it to represent a single experiment.

Acknowledgments. The authors express their thanks to $M$. Schurter and P. Périsset for their excellent technical support and to all members of the Environmental Physics Department of the Swiss Federal Institute for Environmental Science and Technology (EAWAG) who participated in the field work. A fluorometer was generously provided by the Limnology Department of EAWAG. This work profited from the remarks of two anonymous reviewers. The project was supported by the Swiss National Science Foundation (grants 20$27751.89,20-32700.91$, and 20-36364.92).

\section{References}

Aeschbach-Hertig, W., Helium und Tritium als Tracer für physikalische Prozesse in Seen, Ph.D. dissertation, 272 pp., Eidgenossische Tech. Hochsch., Zurich, 1994.

Batchelor, G. K., The application of the similarity theory of turbulence to atmospheric diffusion, Q.J. R. Meteorol. Soc., 76, 133-146, 1950.

Carter, H. H., and A. Okubo, A study of the physical processes of the movement and dispersion in the Cape Kennedy area. Final report under the U.S. Atomic Energy Commission, Rep. NYO-2973-1, 164 pp., Chesapeake Bay Inst., Johns Hopkins Univ., Baltimore, Md., 1965.

Colman, J. A., and D. E. Armstrong, Horizontal diffusivity in a small, ice-covered lake, Limnol. Oceanogr., 28, 1020-1026, 1983.

Csanady, G. T., Turbulent Diffusion in the Environment, D. Reidel, Norwell, Mass., 1973.

Ewart, T. E., and W. P. Bendiner, An observation of the horizontal and vertical diffusion of a passive tracer in the deep ocean, $J$. Geophys. Res., 86, 10,974-10,982, 1981.

Foxworthy, J. F., R. B. Tibby, and G. M. Barsom, Dispersion of a surface waste field in the sea, J. Water Pollut. Control Fed., 38, 1170-1193, 1966.

Gargett, A. E., Vertical eddy diffusivity in the ocean interior, J. Mar. Res., 42, 359-393, 1984.

Gregg, M. C., Diapycnal mixing in the seasonal thermocline: A review, J. Geophys. Res., 92, 5249-5286, 1987.

Gregg, M. C., Scaling turbulent dissipation in the thermocline, J. Geophys. Res., 94, 9686-9698, 1989.

Imboden, D. M., and A. Wüest, Mixing mechanisms in lakes, in Physics and Chemistry of Lakes, edited by A. Lerman, D. Imboden, and J. Gat, pp. 83-138, Springer-Verlag, New York, 1995.

Joseph, J., and H. Sendner, Über die horizontale Diffusion im Meere, Dtsch. Hydrogr. Z., 11, 49-77, 1958.

Kawai, H., Scale dependence of divergence and vorticity of near surface flows in the sea, 2, Results and interpretations, J. Oceanogr. Soc. Jpn., 41, 167-175, 1985.

Kolmogorov, N. A., Dissipation of energy under locally isotropic turbulence, Dokl. Akad. Nauk SSSR, 30, 16-18, 1941.

Kullenberg, G., Apparent horizontal diffusion in stratified vertical shear flow, Tellus, 24, 17-28, 1972.

Ledwell, J. R., and A. J. Watson, The Santa Monica Basin tracer experiment: A study of diapycnal and isopycnal mixing, J. Geophys. Res., 96, 8695-8718, 1991.

Ledwell, J. R., A. J. Watson, and C. L. Law, Evidence of slow mixing across the pycnocline from an open-ocean tracer-release experiment, Nature, 364, 701-703, 1993.

Li, Y. H., Vertical eddy diffusion coefficient in Lake Zürich, Schweiz. Z. Hydrol., 35, 1-7, 1973.

Monin, A. S., and R. V. Ozmidov, Turbulence in the Ocean, D. Reidel, Norwell, Mass., 1985.

Murthy, C. R., Horizontal diffusion characteristics in Lake Ontario, $J$. Phys. Oceanogr., 6, 76-84, 1976.

Oakey, N. S., Determination of the rate of dissipation of turbulent energy from simultaneous temperature and velocity shear microstructure measurements, J. Phys. Oceanogr., 12, 256-271, 1982.

Okubo, A., The effect of shear in an oscillatory current on horizontal diffusion from an instantaneous source, Oceanol. Limnol., 1, 194204, 1967. 
Okubo, A., Some remarks on the importance of the "shear effect" on horizontal diffusion, J. Oceanogr. Soc. Jpn., 24, 60-69, 1968.

Okubo, A., Oceanic diffusion diagrams, Deep Sea Res., 18, 789-802, 1971.

Osborn, T. R., and C. S. Cox, Oceanic fine structure, Geophys. Astrophys. Fluid Dyn., 3, 321-345, 1972.

Pal, B. K., and B. G. Sanderson, Measurements of drifter cluster dispersion, Atmos. Ocean, 30, 621-651, 1992.

Palmer, M. D., R. Jarvis, and L. Thompson, Drogue-cluster and dyedispersion measurements, Can. J. Civ. Eng., 14, 320-326, 1987.

Peeters, F., Horizontale Mischung in Seen, Ph.D. dissertation, 147 pp., Eidgenossische Tech. Hochsch., Zurich, 1994.

Powell, T., and A. Jassby, The estimation of vertical eddy diffusivities below the thermocline in lakes, Water Resour. Res., 10, 191-198, 1974.

Quay, P. D., W. S. Broecker, E. H. Hesslein, E. J. Fee, and D. W. Schindler, Whole lake tritium spikes to measure horizontal and vertical mixing rates, in Isotopes in Lake Studies, pp. 175-194, Int. At. Energy Agency, Vienna, 1979.

Sanderson, B. G., and D. A. Booth, The fractal dimension of drifter trajectories and estimates of horizontal eddy-diffusivity, Tellus Ser. $A, 43,334-349,1991$.

Sanderson, B. G., and A. Okubo, Diffusion by internal waves, J. Geophys. Res., 93, 3570-3582, 1988.

Sanderson, B. G., A. Okubo, I. T. Webster, S. Kioroglou, and R. Appeldoorn, Observations and idealized models of dispersion on the Southwestern Ouerto Rican Insular Shelf, Math. Comput. Model., 21(6), 39-63, 1995.

Smart, P. L., and I. M. S. Laidlaw, An evaluation of some fluorescent dyes for water tracing, Water Resour. Res., 13, 15-33, 1977.

Taylor, G. I., Diffusion by continuous movements, Proc. London Math Soc., 20, 196-211, 1921.

Ward, G. H., Jr., Dye diffusion experience in the Texas bays: Low-wave conditions, J. Geophys. Res., 90, 4959-4968, 1985.

Wüest, A., D. C. Van Senden, J. Imberger, G. Piepke, and M. Gloor, Comparison of diapycnal diffusivity measured by tracer and microstructure techniques, Dyn. Atmos. Oceans, 24, 27-39, 1996.

Yanagi, T., K. Murashita, and H. Higuchi, Horizontal turbulent diffusivity in the sea, Deep Sea Res., Part A, 29, 217-226, 1982.

Young, W. R., P. B. Rhines, and C. J. R. Garrett, Shear-flow, internal waves and horizontal mixing in the ocean, J. Phys. Oceanogr., 12, 515-527, 1982.

D. M. Imboden, F. Peeters, G. Piepke, and A. Wüest, Swiss Federal Institute for Environmental Science and Technology and Swiss Federal Institute of Technology, Ueberlandstrasse 133, CH-8600, Dübendorf, Switzerland. (e-mail: peeters@hermes.eawag.ch)

(Received May 8, 1995; revised December 22, 1995; accepted March 19, 1996.) 